\title{
Infrared excess around nearby red giant branch stars and Reimers law ${ }^{\star}$
}

\author{
M. A. T. Groenewegen
}

Koninklijke Sterrenwacht van België, Ringlaan 3, 1180 Brussel, Belgium

e-mail: marting@oma.be

Received 17 October 2011 / Accepted 30 January 2012

\section{ABSTRACT}

\begin{abstract}
Context. Mass loss is one of the fundamental properties of asymptotic giant branch (AGB) stars, but for stars with initial masses below $\sim 1 M_{\odot}$, the mass loss on the first red giant branch (RGB) actually dominates mass loss on the AGB. Nevertheless, mass loss on the RGB is still often parameterised by a simple Reimers law in stellar evolution models.

Aims. We study the infrared excess and mass loss of a sample of nearby RGB stars with reliably measured HiPPARcos parallaxes and compare the mass loss to that derived for luminous stars in clusters.

Methods. The spectral energy distributions of a well-defined sample of 54 RGB stars are constructed, and fitted with the dust radiative transfer model DUSTY. The central stars are modelled by MARCS model atmospheres. In a first step, the best-fit MARCS model is derived, basically determining the effective temperature. In a second step, models with a finite dust optical depth are fitted and it is determined whether the reduction in $\chi^{2}$ in such models with one additional free parameter is statistically significant.

Results. Among the 54 stars, 23 stars are found to have a significant infrared excess, which is interpreted as mass loss. The most luminous star with $L=1860 L_{\odot}$ is found to undergo mass loss, while none of the 5 stars with $L<262 L_{\odot}$ display evidence of mass loss. In the range $265<L<1500 L_{\odot}, 22$ stars out of 48 experience mass loss, which supports the notion of episodic mass loss. It is the first time that excess emission is found in stars fainter than $\sim 600 L_{\odot}$. The dust optical depths are translated into massloss rates assuming a typical expansion velocity of $10 \mathrm{~km} \mathrm{~s}^{-1}$ and a dust-to-gas ratio of 0.005 . In this case, fits to the stars with an excess result in $\log \dot{M}\left(M_{\odot} \mathrm{yr}^{-1}\right)=(1.4 \pm 0.4) \log L+(-13.2 \pm 1.2)$ and $\log \dot{M}\left(M_{\odot} \mathrm{yr}^{-1}\right)=(0.9 \pm 0.3) \log (L R / M)+(-13.4 \pm 1.3)$ assuming a mass of $1.1 M_{\odot}$ for all objects. We caution that if the expansion velocity and dust-to-gas ratio have different values from those assumed, the constants in the fit will change. If these parameters are also functions of luminosity, then this would affect both the slopes and the offsets. The mass-loss rates are compared to those derived for luminous stars in globular clusters, by fitting both the infrared excess, as in the present paper, and the chromospheric lines. There is excellent agreement between these values and the mass-loss rates derived from the chromospheric activity. There is a systematic difference with the literature mass-loss rates derived from modelling the infrared excess, and this has been traced to technical details on how the DUSTY radiative transfer model is run. If the present results are combined with those from modelling the chromospheric emission lines, we obtain the fits $\log \dot{M}$ $\left(M_{\odot} \mathrm{yr}^{-1}\right)=(1.0 \pm 0.3) \log L+(-12.0 \pm 0.9)$ and $\log \dot{M}\left(M_{\odot} \mathrm{yr}^{-1}\right)=(0.6 \pm 0.2) \log (L R / M)+(-11.9 \pm 0.9)$, and find that the metallicity dependence is weak at best. The predictions of these mass-loss rate formula are tested against the recent RGB mass loss determination in NGC 6791. Using a scaling factor of $\sim 10 \pm \sim 5$, both relations can fit this value. That the scaling factor is larger than unity suggests that the expansion velocity and/or dust-to-gas ratio, or even the dust opacities, are different from the values adopted. Angular diameters are presented for the sample. They may serve as calibrators in interferometric observations.
\end{abstract}

Key words. circumstellar matter - stars: fundamental parameters - stars: mass-loss - planetary systems

\section{Introduction}

Almost all stars with masses between 1 and $8 M_{\odot}$ pass through the asymptotic giant branch (AGB). On the AGB, the mass-loss rate exceeds the nuclear burning rate, implying that the massloss process dominates stellar evolution. Although not understood in all its details, the relevant process is believed to be that of pulsation-enhanced dust-driven winds: shock waves created by stellar pulsation lead to a dense, cool, extended stellar atmosphere, allowing for efficient dust formation. The grains are accelerated away from the star by radiation pressure, dragging the gas along (see the various contributions in Habing \& Olofsson 2003, for an overview). Following the advent of Spitzer, an analysis of 200 carbon- and oxygen-rich AGB stars in the Small and Large Magellanic Clouds with Spitzer IRS spectra show a clear relation between the mass-loss rate and both the pulsation

^ Appendix A and Table 4 are available in electronic form at http://www. aanda.org period and the luminosity (Groenewegen et al. 2009, and references therein), confirming earlier work on Galactic stars.

The focus of the present paper however is mass loss on the first red giant branch (RGB). For stars with initial masses of $\$ 2.2 M_{\odot}$, this is a prominent evolutionary phase where stars reach high luminosities $\left(\log \left(L / L_{\odot}\right) \sim 3\right)$. Low- and intermediatemass stars must lose about $0.2 M_{\odot}$ on the RGB in order to explain the morphology on the horizontal branch (e.g. Catelan 2000, and references therein) and the pulsation properties of RR Lyrae stars (e.g. Caloi \& d'Antona 2008). For the lowest initial masses $\left(\lesssim 1 M_{\odot}\right.$ ), the total mass lost on the RGB dominates that of the AGB phase, and therefore it is equally important to understand how this process develops. In stellar evolutionary models, the RGB mass loss is often parameterised by the Reimers law (1975) with some scaling parameter (typically $\eta \sim 0.4)$.

The RGB mass loss can arise from chromospheric activity (see Mauas et al. 2006; Mészáros et al. 2009; Vieytes et al. 2011) or can also be pulsation-enhanced and dust-driven. The studies 
of Boyer et al. (2010), Origlia et al. (2007, 2010), and Momany et al. (2012) of 47 Tuc, and McDonald et al. $(2009,2011)$ of $\omega$ Cen illustrate the current state of affairs regarding the dust modelling. Boyer et al. (2010) uses Spitzer 3.6 and $8 \mu \mathrm{m}$ data to show that the reddest colours on the RGB are reached at the tip of the RGB (TRGB), and that these are known long period variables (Clement et al. 2001; Lebzelter \& Wood 2005) with periods between 50 and 220 days. In McDonald et al. (2009), optical and NIR data is combined with Spitzer IRAC and MIPS $24 \mu \mathrm{m}$ data to model the spectral energy distributions (SEDs) and derive mass-loss rates. They conclude that two-thirds of the total mass loss is by dusty winds and one-third by chromospheric activity. They show that the highest (dust) mass-loss rates occur near the TRGB, which, indeed, are known variable stars being mostly semi-regular pulsators. At lower luminosities along the $\mathrm{RGB}$, there is an excess at $24 \mu \mathrm{m}$ that can be translated into a mass-loss rate but the uncertainties are large. In an alternative approach, using asteroseismology to estimate the mass of red clump stars, and stars on the RGB fainter than the luminosity of the clump, Miglio et al. (2012) estimate the amount of mass lost in NGC 6791 and NGC 6819, and conclude that it is consistent with a Reimers law with $0.1 \lesssim \eta \lesssim 0.3$.

In the present paper, a complimentary approach is taken by studying the infra-red excess around nearby RGB stars, based on a sample of stars with accurate parallaxes. In Sect. 2, we present the sample in addition to the photometric data used to constrain the modelling. In Sect. 3, the dust radiative transfer models are introduced, and the results are presented in Sect. 4. Our results are discussed in Sect. 5.

\section{The sample}

We selected our sample from the HiPPARcos catalog. The parallaxes were taken from van Leeuwen (2007), and other data for the stars was gathered from the original release (ESA 1997). In a first step, supposedly single stars were selected where the parameter fit was good (Hipparcos flags isoln $=5$ and gof $<5.0$ ). To ensure an accurate determination of the luminosity, a positive parallax and relative error of smaller than $10 \%$ were required $\left(\pi>0, \sigma_{\pi} / \pi<0.1\right)$.

It is well-established (see e.g. McDonald et al. 2009) that mass loss is larger in stars near the tip of the RGB but also that these stars often show variability. To illustrate this, Fig. 1 shows the fraction of variable stars across the Hertzsprung-Russell diagram. We plot 44177 stars from HIPPARcos that have a parallax error of smaller than $15 \%$ and an error in $(V-I)$ of smaller than $0.15 \mathrm{mag}$. The cells have a width of $0.1 \mathrm{mag}$ in $(V-I)$ and $0.25 \mathrm{mag}$ in $M_{V}$. The RGB is clearly visible with a very high fraction of variables. On the basis of this, a further selection of $(V-I)>1.5$ (and $\left.\sigma_{(V-I)}<0.1\right)$ was imposed. The possible effect of using this lower limit to the $(V-I)$ colour on the fraction of mass-losing RGB stars is discussed in Sect. 5.1.

At this point in the selection, the interstellar reddening $A_{V}$ is determined using the method outlined in Groenewegen (2008), which combines various three-dimensional estimates of $A_{V}$ (Marshall et al. 2006; Drimmel et al. 2003; Arenou et al. 1992).

Following Koen \& Laney (2000; see also Dumm \& Schild 1998), the effective temperature is estimated from the relation $\log T_{\text {eff }}(\mathrm{K})=3.65-0.035 \cdot(V-I)_{0}$ and the stellar radius (in solar units) from $\log R=2.97-\log (\pi)-0.2 \cdot\left(V_{\mathrm{o}}+0.356 \cdot(V-I)_{0}\right)$, from which the luminosity in solar units is then determined. A final selection using $M_{V}<+1.0$ (see Fig. 1), $100<L<2000 L_{\odot}$, and $A_{V}<0.1$ is applied, again to ensure the selection of giants

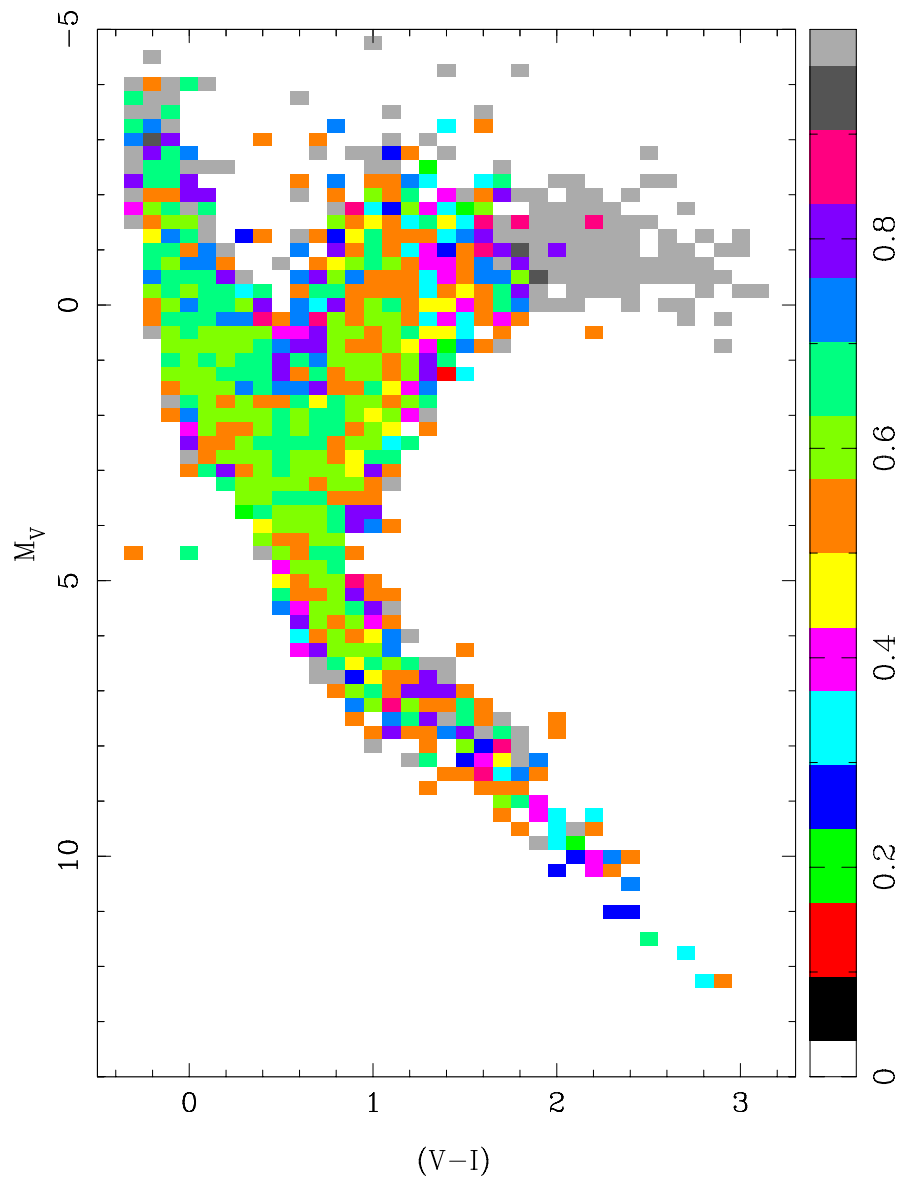

Fig. 1. The fraction of variable stars in the Hipparcos catalog based on the van Leeuwen (2007) parallax data. The bin size is 0.1 in $(V-I)$ and 0.25 in $M_{V}$. Considered are stars with an relative parallax error $<0.15$ and an error in $(V-I)<0.15 \mathrm{mag}$. The RGB stands out as having almost $100 \%$ variability.

and that reddening will play no role in the interpretation of the results.

In the section below the methodology is outlined but is based on fitting models to the SED. Therefore, the availability of sufficient photometric data is crucial, especially in the optical and near-infrared as this will be the main constraint in deriving the best-fit model atmosphere. For this reason, stars that lacked either optical $U B V$ and/or $J H K$ data were removed from the sample. In addition, stars that have an IRAS CIRR3 flag > $25 \mathrm{MJy} / \mathrm{sr}$ were also removed, in order to avoid contamination by cirrus of the IRAS 60 and $100 \mu \mathrm{m}$ data.

The sample thus selected contains 54 objects ( 15 of spectral type $\mathrm{K}$, and $39 \mathrm{M}$ giants) of which the basic properties have been listed in Table 1.

Most data listed come from the HipParcos catalog, including the type of variability and the difference between the $95 \%$ and $5 \%$ percentile values of $H_{\mathrm{P}}\left(\Delta H_{\mathrm{P}}\right)$. In addition, the reddening (see above, Col. 4), the spectral type (from SIMBAD, Col. 7), and both $[\mathrm{Fe} / \mathrm{H}]$ and $\log g$ determinations from the literature (Cols. 10, 11) are listed.

\section{The model}

The code used in this paper is based on that presented by Groenewegen et al. (2009). In that paper, a dust radiative transfer model was included as a subroutine in a minimisation code using 
M. A. T. Groenewegen: Infrared excess around nearby red giant branch stars and Reimers law

Table 1. The RGB sample.

\begin{tabular}{|c|c|c|c|c|c|c|c|c|c|c|}
\hline HIP & $\begin{array}{l}\text { Parallax } \\
\text { (mas) }\end{array}$ & $M_{V}$ & $A_{V}$ & V & $(V-I)$ & Spectral type & $\begin{array}{l}\text { Variability } \\
\text { type }^{a}\end{array}$ & $\begin{array}{c}\Delta H_{\mathrm{P}} \\
(\mathrm{mag})\end{array}$ & {$[\mathrm{Fe} / \mathrm{H}]$} & $\log g^{b}$ \\
\hline 4147 & $5.56 \pm 0.22$ & -1.58 & 0.09 & 4.78 & 1.66 & M0III & $\mathrm{M}$ & 0.03 & & \\
\hline 12107 & $7.00 \pm 0.42$ & -0.33 & 0.08 & 5.53 & 1.98 & MOIII & $\mathrm{U}$ & 0.05 & & \\
\hline 32173 & $10.08 \pm 0.33$ & -0.01 & 0.06 & 5.04 & 1.50 & K5III & $\mathrm{C}$ & 0.02 & -0.03 & $1.5(1)$ \\
\hline 37300 & $6.21 \pm 0.29$ & -1.07 & 0.07 & 5.04 & 1.72 & MOIII & $\mathrm{U}$ & 0.03 & & \\
\hline 37946 & $7.51 \pm 0.41$ & -0.55 & 0.08 & 5.15 & 2.03 & M3III & $\mathrm{U}$ & 0.04 & & \\
\hline 41822 & $7.88 \pm 0.30$ & -0.24 & 0.05 & 5.33 & 1.57 & K5III & $\mathrm{U}$ & 0.03 & & \\
\hline 44126 & $5.33 \pm 0.44$ & -0.16 & 0.09 & 6.30 & 2.48 & M4III & $\mathrm{U}$ & 0.10 & & \\
\hline 44390 & $10.36 \pm 0.25$ & -0.23 & 0.04 & 4.74 & 2.15 & M3III & $\mathrm{U}$ & 0.04 & & \\
\hline 44857 & $6.25 \pm 0.30$ & -0.93 & 0.06 & 5.15 & 1.55 & K5III & M & 0.03 & -0.23 & $1.66(2)$ \\
\hline 46750 & $9.92 \pm 0.18$ & -0.74 & 0.04 & 4.32 & 1.63 & K5III & M & 0.02 & -0.29 & $1.6(2)$ \\
\hline 47723 & $5.35 \pm 0.33$ & -1.08 & 0.08 & 5.36 & 1.94 & M2III & $\mathrm{U}$ & 0.06 & & \\
\hline 49005 & $6.51 \pm 0.35$ & -0.49 & 0.06 & 5.50 & 1.51 & K5III & M & 0.02 & & \\
\hline 49029 & $8.04 \pm 0.29$ & -0.85 & 0.05 & 4.68 & 1.96 & M2III & $\mathrm{U}$ & 0.03 & & \\
\hline 52366 & $4.02 \pm 0.33$ & -1.04 & 0.08 & 6.02 & 2.45 & M2III & $\mathrm{U}$ & 0.09 & & \\
\hline 52863 & $5.99 \pm 0.42$ & -0.26 & 0.06 & 5.92 & 1.91 & M2III & M & 0.04 & & \\
\hline 53449 & $8.42 \pm 0.37$ & +0.49 & 0.05 & 5.91 & 3.50 & M5.5I & $\mathrm{P}$ & 0.26 & & $2.3(1)$ \\
\hline 53726 & $3.96 \pm 0.38$ & -1.10 & 0.08 & 5.99 & 2.17 & M2III & $\mathrm{U}$ & 0.06 & & \\
\hline 53907 & $5.57 \pm 0.24$ & -1.61 & 0.07 & 4.73 & 1.77 & MOIII & $\mathrm{U}$ & 0.03 & -0.23 & $1.22(3)$ \\
\hline 54537 & $5.96 \pm 0.50$ & -0.31 & 0.08 & 5.89 & 2.16 & M2III & $\mathrm{U}$ & 0.06 & & \\
\hline 55687 & $8.67 \pm 0.22$ & -0.55 & 0.05 & 4.81 & 1.67 & K5III & M & 0.03 & -0.38 & $1.61(2)$ \\
\hline 56127 & $5.38 \pm 0.31$ & -1.64 & 0.07 & 4.77 & 1.62 & K3.5I & M & 0.03 & -0.31 & $1.80(3)$ \\
\hline 56211 & $9.80 \pm 0.16$ & -1.27 & 0.04 & 3.82 & 1.79 & MOIII & $\mathrm{U}$ & 0.05 & & \\
\hline 60122 & $5.51 \pm 0.28$ & -1.08 & 0.07 & 5.28 & 1.90 & MOIII & $\mathrm{U}$ & 0.04 & & \\
\hline 60795 & $7.82 \pm 0.32$ & +0.09 & 0.06 & 5.68 & 1.88 & M2III & M & 0.03 & & \\
\hline 61658 & $6.64 \pm 0.31$ & -0.29 & 0.08 & 5.68 & 2.32 & M3III & $\mathrm{U}$ & 0.07 & & \\
\hline 62443 & $5.59 \pm 0.45$ & +0.08 & 0.08 & 6.42 & 2.14 & M4III & $\mathrm{U}$ & 0.05 & & \\
\hline 63355 & $10.06 \pm 0.28$ & -0.28 & 0.06 & 4.76 & 1.79 & M1III & M & 0.03 & & $1.0(1)$ \\
\hline 64607 & $6.82 \pm 0.32$ & -0.26 & 0.07 & 5.64 & 1.53 & MOIII & $\mathrm{U}$ & 0.05 & & \\
\hline 66417 & $7.12 \pm 0.34$ & -0.09 & 0.07 & 5.72 & 1.96 & M2III & $\mathrm{U}$ & 0.05 & & \\
\hline 66738 & $6.23 \pm 0.22$ & -1.47 & 0.07 & 4.63 & 1.97 & M2III & $\mathrm{U}$ & 0.06 & +0.30 & $1.60(4)$ \\
\hline 67605 & $4.80 \pm 0.38$ & -0.79 & 0.09 & 5.89 & 1.94 & M2III & $\mathrm{U}$ & 0.05 & & \\
\hline 67627 & $8.79 \pm 0.20$ & -0.75 & 0.05 & 4.58 & 2.35 & M3.5I & $\mathrm{U}$ & 0.09 & -0.24 & $1.25(3)$ \\
\hline 67665 & $5.43 \pm 0.20$ & -1.65 & 0.08 & 4.76 & 1.63 & K5III & $\mathrm{U}$ & 0.12 & +0.50 & $0.50(2)$ \\
\hline 69068 & $5.95 \pm 0.25$ & -0.94 & 0.08 & 5.26 & 1.97 & M1.5I & $\mathrm{U}$ & 0.08 & & \\
\hline 69373 & $7.62 \pm 0.19$ & -0.46 & 0.05 & 5.18 & 1.93 & M2III & $\mathrm{U}$ & 0.03 & & \\
\hline 71280 & $3.82 \pm 0.26$ & -1.44 & 0.09 & 5.74 & 1.71 & M1III & $\mathrm{U}$ & 0.04 & & \\
\hline 73568 & $8.78 \pm 0.28$ & -0.55 & 0.07 & 4.80 & 1.54 & K4III & M & 0.02 & -0.04 & $1.68(2)$ \\
\hline 76307 & $5.26 \pm 0.24$ & -1.35 & 0.10 & 5.14 & 1.90 & M1.5I & $\mathrm{U}$ & 0.05 & & \\
\hline 77661 & $8.72 \pm 0.30$ & -0.62 & 0.06 & 4.74 & 1.60 & K5III & M & 0.03 & -0.17 & $1.68(2)$ \\
\hline 78632 & $3.40 \pm 0.33$ & -1.24 & 0.09 & 6.19 & 1.87 & M1III & $\mathrm{U}$ & 0.04 & & \\
\hline 80042 & $4.32 \pm 0.43$ & -0.34 & 0.09 & 6.57 & 1.57 & M2III & $\mathrm{U}$ & 0.06 & & \\
\hline 80197 & $5.08 \pm 0.22$ & -1.37 & 0.10 & 5.20 & 2.00 & M2III & $\mathrm{U}$ & 0.05 & & \\
\hline 80214 & $5.48 \pm 0.24$ & -1.00 & 0.09 & 5.40 & 1.57 & K5III & M & 0.03 & -0.16 & $1.76(2)$ \\
\hline 82073 & $9.21 \pm 0.41$ & -0.10 & 0.07 & 5.15 & 1.54 & K5III & M & 0.03 & -0.03 & $1.52(3)$ \\
\hline 83430 & $8.26 \pm 0.26$ & -0.52 & 0.07 & 4.97 & 2.08 & M3III & $\mathrm{U}$ & 0.05 & & \\
\hline 84835 & $5.84 \pm 0.19$ & -0.75 & 0.10 & 5.51 & 1.71 & MOIII & M & 0.04 & & \\
\hline 87833 & $21.15 \pm 0.10$ & -1.17 & 0.03 & 2.24 & 1.54 & K5III & M & 0.02 & -0.05 & $1.32(3)$ \\
\hline 88122 & $5.71 \pm 0.25$ & -0.63 & 0.10 & 5.69 & 1.69 & MOIII & M & 0.03 & & \\
\hline 98401 & $6.20 \pm 0.31$ & +0.08 & 0.08 & 6.20 & 2.09 & M3III & $\mathrm{U}$ & 0.06 & & \\
\hline 106140 & $8.29 \pm 0.19$ & -0.97 & 0.08 & 4.52 & 1.82 & M1III & $\mathrm{U}$ & 0.04 & & \\
\hline 112716 & $10.28 \pm 0.29$ & -0.97 & 0.08 & 4.05 & 1.72 & K5III & M & 0.03 & & \\
\hline 114144 & $9.92 \pm 0.29$ & -0.54 & 0.06 & 4.54 & 1.79 & M1III & $\mathrm{U}$ & 0.04 & & $1.0(1)$ \\
\hline 115669 & $11.50 \pm 0.22$ & -0.38 & 0.06 & 4.38 & 1.52 & K4III & M & 0.02 & -0.20 & $1.66(2)$ \\
\hline 117718 & $7.07 \pm 0.27$ & -0.79 & 0.10 & 5.06 & 2.09 & M2III & $\mathrm{U}$ & 0.06 & & \\
\hline
\end{tabular}

Notes. ${ }^{(a)}$ Variability type, Field H52 in the HiPparcos (ESA 1997) catalog. Meaning: C = "constant", not detected as being variable, D = duplicityinduced variability flag, $\mathrm{M}=$ possible micro variable, $\mathrm{U}=$ unsolved variable, $\mathrm{P}=$ Periodic, $\mathrm{R}=$ revised colour index. ${ }^{(b)} \mathrm{References}$ for $[\mathrm{Fe} / \mathrm{H}]$ and $\log$ g: (1) Massarotti et al. (2008, and references therein); (2) McWilliam (1990); (3) Cenarro et al. (2007); (4) Fernández-Villacañas et al. (1990). 
the the MRQMIN routine (using the Levenberg-Marquardt method from Press et al. 1992). The parameters that were fitted in the minimisation process include the dust optical depth in the $V$ band $\left(\tau_{V}\right)$, luminosity, and the dust temperature at the inner radius $\left(T_{\mathrm{c}}\right)$. The output of a model is an SED, which is folded with the relevant filter response curves to produce magnitudes that can be compared to the observations (see Groenewegen 2006). Spectra can also be included in the minimisation process. In Groenewegen et al. (2009) the dust radiative transfer model was that of Groenewegen (1993; also see Groenewegen 1995), but this has since been replaced by the dust radiative transfer model DUSTY (Ivezić et al. 1999).

The central star was modelled by a MARCS stellar photosphere model $^{1}$ (Gustafsson et al. 2008). Models for temperatures between $3200 \mathrm{~K}$ and $4000 \mathrm{~K}$ (in steps of $100 \mathrm{~K}$ ), and those of $4250 \mathrm{~K}$ and $4500 \mathrm{~K}$, with solar metallicity and $\log g=1.5$ were considered. Spectroscopically determined metallicites and gravities were only available for a third of the sample (Table 1) but indicate that $\log g=1.5$ is an appropriate value. The median metallicity is -0.17 dex, so slightly subsolar.

As the mass-loss rates in RGB stars are expected to be small, and the IRAS LRS spectra (see below) show no hint of a $9.8 \mu \mathrm{m}$ silicate feature, two types of dust were considered: aluminium oxide (AlOx), and metallic iron. The first species is expected as one of the first condensates in an oxygen-rich environment (see Niyogi et al. 2011, for a recent discussion), while metallic iron has gained interest in the past few years as a source of opacity (see e.g. McDonald et al. 2010).

Absorption and scattering coefficients were calculated for grains of radius $0.15 \mu \mathrm{m}$ in the approximation of a "distribution of hollow spheres" (DHS) (Min et al. 2005, a vacuum fraction of $70 \%$ is adopted) using the optical constants of Begemann et al. (1997) for AlOx, and Pollack et al. (1994) for iron.

The SEDs were constructed considering the following sources of photometry: Mermilliod (1991) for $U B V$ photometry, and the $I$ magnitude as listed in the Hipparcos catalog, Gezari et al. (1999) for JHKLM photometry (2MASS was not considered, as owing to the brightness of the sources, the 2MASS photometry was either saturated or had very large error bars), the IRAS Point Source Catalog (PSC, Beichman et al. 1985) and Faint Source Catalog (FSC, Moshir et al. 1989) for 12, 25, 60, and $100 \mu \mathrm{m}$ data (only data with flux-quality 3 were considered), AKARI IRC (Ishihara et al. 2010) and FIS (Yamamura et al. 2010) mid- and far-IR data, In addition, the IRAS LRS spectra (Olnon et al. 1986) available from Volk \& Cohen $(1989)^{2}$ were used when available. The spectra were typically scaled by factors $1.3-1.6$ to ensure that they agreed with the IRAS $12 \mu \mathrm{m}$ and/or AKARI S9W filter.

In a first iteration, models with effectively no mass loss $\left(\tau_{V}=10^{-5}\right)$ were run by varying only the effective temperature. A $r^{-2}$ density distribution was assumed, and the condensation temperature was fixed at $1000 \mathrm{~K}$ in all models, as this cannot be constrained from the current photometric datasets for low mass-loss rates. The best-fit model was determined. In a second iteration, for that effective temperature, models with fixed optical depths of $\tau_{V}=10^{-4}, 10^{-3}, 10^{-2}$ were run for both iron and AlOx dust. The best-fit model was determined (thus fixing the dust component), and then models where $\tau$ was also allowed to vary were run. Finally, models were run in which $\tau$ was allowed to vary using effective temperatures one step cooler and hotter in the available grid of MARCS models.

\footnotetext{
${ }^{1}$ http://marcs.astro.uu.se/

${ }^{2}$ http://www.iras.ucalgary.ca/ volk/getlrs_plot.html
}

\section{Results}

Table 2 lists the parameters of the models that provide the best fit to the observed data. The fit error in the derived optical depth is typically small, in the median only $5 \%$, but in some cases much larger. However, this error does not take into account e.g. the effect of varying the model atmosphere. Some tests were performed by varying the gravity of the model atmosphere by \pm 0.5 dex and the metallicity by \pm 0.25 dex and refitting the optical depth. The results suggest that this represents an additional $50 \%$ uncertainty. The largest uncertainty is in the conversion from optical depth to mass-loss rate. On the one hand, a systematic error as the mean velocity and mean dust-to-gas ratio may differ from the adopted values, and, on the other hand, the values for individual stars will scatter around these mean values. A random error of a factor of two is adopted in the latter case, and this error dominates the error budget.

Examples of the fits are shown in Fig. 2, and all fits are displayed in Appendix A. The panels with the SEDs show the best fit (solid line), and a model without mass loss (dashed line) for comparison. The differences are often small, certainly visually, but are statistically significant.

From inspecting the plots, it is also clear that far-IR data $(\sim 80-200 \mu \mathrm{m})$ would certainly be very valuable in constraining the models, as any excess is expected to be largest in that wavelength region. In this respect, it is unfortunate that the AKARI/FIS has relatively poor sensitivity. All 54 stars have AKARI/IRC S9W and L18W data, but only 15 have FIS WSband data at $90 \mu \mathrm{m}$ and none of the stars are detected in the filters at 140 and $160 \mu \mathrm{m}$. None of the stars are detected in the Planck Early Release Compact Source Catalogue (Planck Collaboration 2011), no appear to have MIPS or Herschel data.

In addition, high-quality mid-IR spectra would also be useful to improve upon the, in most cases, relatively poor quality IRAS LRS spectrum. Only for one object does an ISO SWS spectrum exist (HIP $87833=\gamma$ Dra). With the current data, no clear dust feature is visible in any of the stars, hence, when a significant infrared excess detected, the best fit is provided by the featureless metallic iron model rather than the aluminium oxide one (except in one case).

Other mechanisms can produce an infrared excess that is not due to dust emission, as discussed in MacDonald et al. (2010), e.g. free-free emission or emission from shells of molecular gas (a MOLsphere; e.g. Tsuji 2000). Even featureless dust emission could in principle also be due to extremely large $(\sim 50 \mu \mathrm{m})$ silicate or amorphous carbon grains (MacDonald et al. 2010), but these species are not really expected to condense and form first in these low-density oxygen-rich CSEs.

Free-free emission is ruled out by MacDonald et al. (2010) as an important source of emission in their sample of giants in $\omega$ Cen. A MOLsphere could be due to many molecules but would most likely manifest itself by the presence of water lines in the 6-8 $\mu \mathrm{m}$ region. This region is not covered by the LRS spectrum so it is impossible to verify this idea directly. MacDonald et al. (2010) studied the effect of using Spitzer IRS data and found that no reasonable combination of column density and temperature could reproduce the flatness of their spectra. For red supergiants, which are much more luminous that RGB stars but that have similar effective temperatures than the stars under study, Verhoelst et al. $(2006,2009)$ found that a MOLsphere alone cannot explain the excess emission in the mid-IR and that a additional source of opacity was needed. 
M. A. T. Groenewegen: Infrared excess around nearby red giant branch stars and Reimers law

Table 2. The RGB sample: fit results.

\begin{tabular}{|c|c|c|c|c|c|c|c|c|}
\hline HIP & $\begin{array}{l}T_{\text {eff }} \\
(\mathrm{K})\end{array}$ & Dust & $\begin{array}{c}L \\
\left(L_{\odot}\right)\end{array}$ & $\begin{array}{c}\theta \\
\text { (mas) }\end{array}$ & $\tau_{V}$ & $\begin{array}{c}\dot{M} \\
\left(M_{\odot} \mathrm{yr}^{-1}\right)\end{array}$ & Code & $\chi_{\mathrm{r}}^{2}$ \\
\hline 4147 & 3800 & & $1044 \pm 25$ & $3.85 \pm 0.15$ & $10^{-5}$ & $1.05 \times 10^{-12}$ & 0 & 74 \\
\hline 12107 & 3700 & & $482 \pm 18$ & $3.47 \pm 0.15$ & $10^{-5}$ & $4.88 \times 10^{-13}$ & 0 & 278 \\
\hline 32173 & 3900 & & $219 \pm 09$ & $3.03 \pm 0.13$ & $10^{-5}$ & $4.95 \times 10^{-13}$ & 0 & 228 \\
\hline 37300 & 3800 & & $665 \pm 10$ & $3.43 \pm 0.13$ & $10^{-5}$ & $8.41 \times 10^{-13}$ & 0 & 13 \\
\hline 37946 & 3700 & & $599 \pm 15$ & $4.15 \pm 0.17$ & $10^{-5}$ & $5.44 \times 10^{-13}$ & 0 & 70 \\
\hline 41822 & 3900 & & $259 \pm 11$ & $2.58 \pm 0.11$ & $10^{-5}$ & $5.38 \times 10^{-13}$ & 0 & 189 \\
\hline 44126 & 3600 & iron & $528 \pm 16$ & $2.92 \pm 0.12$ & $(4.99 \pm 0.15) \times 10^{-2}$ & $2.54 \times 10^{-09}$ & 1 & 46 \\
\hline 44390 & 3600 & iron & $522 \pm 14$ & $5.65 \pm 0.23$ & $(8.36 \pm 0.94) \times 10^{-3}$ & $4.21 \times 10^{-10}$ & 1 & 34 \\
\hline 44857 & 3800 & & $560 \pm 20$ & $3.17 \pm 0.13$ & $10^{-5}$ & $7.72 \times 10^{-13}$ & 0 & 202 \\
\hline 46750 & 3800 & & $467 \pm 10$ & $4.59 \pm 0.18$ & $10^{-5}$ & $7.05 \times 10^{-13}$ & 0 & 60 \\
\hline 47723 & 3700 & & $842 \pm 24$ & $3.51 \pm 0.14$ & $10^{-5}$ & $9.21 \times 10^{-13}$ & 0 & 97 \\
\hline 49005 & 4000 & & $293 \pm 04$ & $2.15 \pm 0.08$ & $10^{-5}$ & $5.85 \times 10^{-13}$ & 0 & 8 \\
\hline 49029 & 3700 & iron & $688 \pm 18$ & $4.76 \pm 0.19$ & $(8.04 \pm 0.74) \times 10^{-3}$ & $4.69 \times 10^{-10}$ & 1 & 31 \\
\hline 52366 & 3600 & iron & $1207 \pm 32$ & $3.33 \pm 0.14$ & $(3.56 \pm 0.15) \times 10^{-2}$ & $2.73 \times 10^{-09}$ & 1 & 28 \\
\hline 52863 & 3700 & & $384 \pm 13$ & $2.65 \pm 0.11$ & $10^{-5}$ & $6.22 \times 10^{-13}$ & 0 & 82 \\
\hline 53449 & 3300 & iron & $1346 \pm 44$ & $8.77 \pm 0.40$ & $(5.58 \pm 0.20) \times 10^{-2}$ & $4.41 \times 10^{-09}$ & 1 & 41 \\
\hline 53726 & 3600 & & $1071 \pm 32$ & $3.09 \pm 0.13$ & $10^{-5}$ & $7.20 \times 10^{-13}$ & 0 & 99 \\
\hline 53907 & 3800 & & $1095 \pm 34$ & $3.95 \pm 0.16$ & $10^{-5}$ & $1.08 \times 10^{-12}$ & 0 & 107 \\
\hline 54537 & 3700 & & $410 \pm 16$ & $2.73 \pm 0.12$ & $10^{-5}$ & $6.43 \times 10^{-13}$ & 0 & 178 \\
\hline 55687 & 3900 & iron & $353 \pm 08$ & $3.31 \pm 0.12$ & $(5.62 \pm 5.16) \times 10^{-4}$ & $2.39 \times 10^{-11}$ & 1 & 30 \\
\hline 56127 & 3900 & iron & $975 \pm 55$ & $3.41 \pm 0.16$ & $(8.08 \pm 0.51) \times 10^{-3}$ & $5.72 \times 10^{-10}$ & 1 & 177 \\
\hline 56211 & 3700 & & $959 \pm 16$ & $6.85 \pm 0.27$ & $10^{-5}$ & $9.83 \times 10^{-13}$ & 0 & 32 \\
\hline 60122 & 3700 & & $836 \pm 20$ & $3.60 \pm 0.14$ & $10^{-5}$ & $6.43 \times 10^{-13}$ & 0 & 61 \\
\hline 60795 & 3800 & & $246 \pm 06$ & $2.62 \pm 0.10$ & $10^{-5}$ & $5.12 \times 10^{-13}$ & 0 & 69 \\
\hline 61658 & 3600 & iron & $601 \pm 22$ & $3.88 \pm 0.17$ & $(1.94 \pm 0.088) \times 10^{-2}$ & $1.05 \times 10^{-09}$ & 1 & 70 \\
\hline 62443 & 3700 & & $279 \pm 14$ & $2.11 \pm 0.10$ & $10^{-5}$ & $5.31 \times 10^{-13}$ & 0 & 363 \\
\hline 63355 & 3800 & iron & $324 \pm 12$ & $3.88 \pm 0.16$ & $(4.14 \pm 0.70) \times 10^{-3}$ & $1.67 \times 10^{-10}$ & 1 & 50 \\
\hline 64607 & 3900 & iron & $267 \pm 13$ & $2.26 \pm 0.10$ & $(1.28 \pm 0.061) \times 10^{-2}$ & $4.75 \times 10^{-10}$ & 1 & 129 \\
\hline 66417 & 3700 & & $336 \pm 07$ & $2.95 \pm 0.12$ & $10^{-5}$ & $5.82 \times 10^{-13}$ & 0 & 46 \\
\hline 66738 & 3600 & & $1458 \pm 32$ & $5.67 \pm 0.23$ & $10^{-5}$ & $8.40 \times 10^{-13}$ & 0 & 55 \\
\hline 67605 & 3800 & iron & $545 \pm 13$ & $2.40 \pm 0.09$ & $(1.47 \pm 0.13) \times 10^{-2}$ & $7.72 \times 10^{-10}$ & 1 & 26 \\
\hline 67627 & 3500 & & $1113 \pm 19$ & $7.40 \pm 0.30$ & $10^{-5}$ & $7.26 \times 10^{-13}$ & 0 & 31 \\
\hline 67665 & 3600 & iron & $1895 \pm 73$ & $5.64 \pm 0.24$ & $(3.96 \pm 0.096) \times 10^{-2}$ & $3.81 \times 10^{-09}$ & 1 & 61 \\
\hline 69068 & 3700 & iron & $790 \pm 23$ & $3.78 \pm 0.15$ & $(1.66 \pm 0.059) \times 10^{-2}$ & $1.04 \times 10^{-09}$ & 1 & 61 \\
\hline 69373 & 3700 & iron & $477 \pm 17$ & $3.76 \pm 0.16$ & $(5.18 \pm 0.63) \times 10^{-3}$ & $2.52 \times 10^{-10}$ & 1 & 68 \\
\hline 71280 & 3900 & iron & $818 \pm 76$ & $2.22 \pm 0.13$ & $(1.45 \pm 0.065) \times 10^{-2}$ & $9.42 \times 10^{-10}$ & 1 & 426 \\
\hline 73568 & 4000 & & $318 \pm 09$ & $3.02 \pm 0.11$ & $10^{-5}$ & $6.09 \times 10^{-13}$ & 0 & 101 \\
\hline 76307 & 3800 & iron & $937 \pm 36$ & $3.45 \pm 0.14$ & $(2.60 \pm 0.71) \times 10^{-3}$ & $1.79 \times 10^{-10}$ & 1 & 80 \\
\hline 77661 & 3900 & $\mathrm{AlOx}$ & $358 \pm 06$ & $3.35 \pm 0.12$ & $(5.00 \pm 1.59) \times 10^{-4}$ & $3.16 \times 10^{-11}$ & 1 & 24 \\
\hline 78632 & 3800 & & $844 \pm 18$ & $2.11 \pm 0.08$ & $10^{-5}$ & $9.45 \times 10^{-13}$ & 0 & 29 \\
\hline 80042 & 3900 & iron & $302 \pm 07$ & $1.52 \pm 0.06$ & $(6.69 \pm 0.17) \times 10^{-2}$ & $2.66 \times 10^{-09}$ & 1 & 30 \\
\hline 80197 & 3700 & iron & $1157 \pm 32$ & $3.90 \pm 0.16$ & $(1.50 \pm 0.064) \times 10^{-2}$ & $1.14 \times 10^{-09}$ & 1 & 47 \\
\hline 80214 & 4000 & & $426 \pm 22$ & $2.19 \pm 0.10$ & $10^{-5}$ & $7.06 \times 10^{-13}$ & 0 & 305 \\
\hline 82073 & 3900 & & $224 \pm 05$ & $2.80 \pm 0.11$ & $10^{-5}$ & $5.01 \times 10^{-13}$ & 0 & 63 \\
\hline 83430 & 3700 & iron & $526 \pm 10$ & $4.28 \pm 0.17$ & $(1.71 \pm 0.11) \times 10^{-2}$ & $8.74 \times 10^{-10}$ & 1 & 23 \\
\hline 84835 & 3800 & & $455 \pm 15$ & $2.67 \pm 0.11$ & $10^{-5}$ & $6.96 \times 10^{-13}$ & 0 & 157 \\
\hline 87833 & 3900 & & $602 \pm 04$ & $10.55 \pm 0.38$ & $10^{-5}$ & $8.20 \times 10^{-13}$ & 0 & 4 \\
\hline 88122 & 4000 & iron & $351 \pm 15$ & $2.07 \pm 0.08$ & $(4.11 \pm 0.084) \times 10^{-2}$ & $1.77 \times 10^{-09}$ & 1 & 60 \\
\hline 98401 & 3700 & iron & $285 \pm 13$ & $2.37 \pm 0.10$ & $(5.59 \pm 0.67) \times 10^{-3}$ & $2.10 \times 10^{-10}$ & 1 & 121 \\
\hline 106140 & 3700 & & $682 \pm 12$ & $4.89 \pm 0.19$ & $10^{-5}$ & $8.29 \times 10^{-13}$ & 0 & 33 \\
\hline 112716 & 3900 & & $567 \pm 13$ & $4.98 \pm 0.19$ & $10^{-5}$ & $7.96 \times 10^{-13}$ & 0 & 54 \\
\hline 114144 & 3900 & iron & $373 \pm 08$ & $3.90 \pm 0.15$ & $(7.07 \pm 8.43) \times 10^{-4}$ & $3.10 \times 10^{-11}$ & 1 & 34 \\
\hline 115669 & 4000 & & $262 \pm 04$ & $3.60 \pm 0.13$ & $10^{-5}$ & $3.70 \times 10^{-13}$ & 0 & 10 \\
\hline 117718 & 3600 & & $816 \pm 19$ & $4.82 \pm 0.20$ & $10^{-5}$ & $6.29 \times 10^{-13}$ & 0 & 68 \\
\hline
\end{tabular}

Notes. Listed are, (Col. 2) the effective temperature of the MARCS model, (Col. 3) the dust component that fits best in the case when $\tau_{V} \neq 10^{-5}$, (Col. 4) luminosity and the internal error (that is, the error in the HiPparcos distance is not included), (Col. 5) the angular diameter in mas and the error (based on the error in $L$, and a $70 \mathrm{~K}$ error in $T_{\text {eff }}$ ), (Col. 6) the dust optical depth in the $V$-band and the internal error, and (Col. 7) the corresponding mass-loss rate assuming a constant expansion velocity of $10 \mathrm{~km} \mathrm{~s}^{-1}$, a dust-to-gas $(\Psi)$ ratio of 0.005 and grain specific density, $\rho$, of $5.1 \mathrm{~g} \mathrm{~cm}^{-3}$ (appropriate for iron grains in a DHS with 70\% vacuum), (Col. 8) a code if the optical depth was fitted (1) or fixed (0), (Col. 9) the reduced $\chi^{2}$ to indicate the goodness of the fit. 

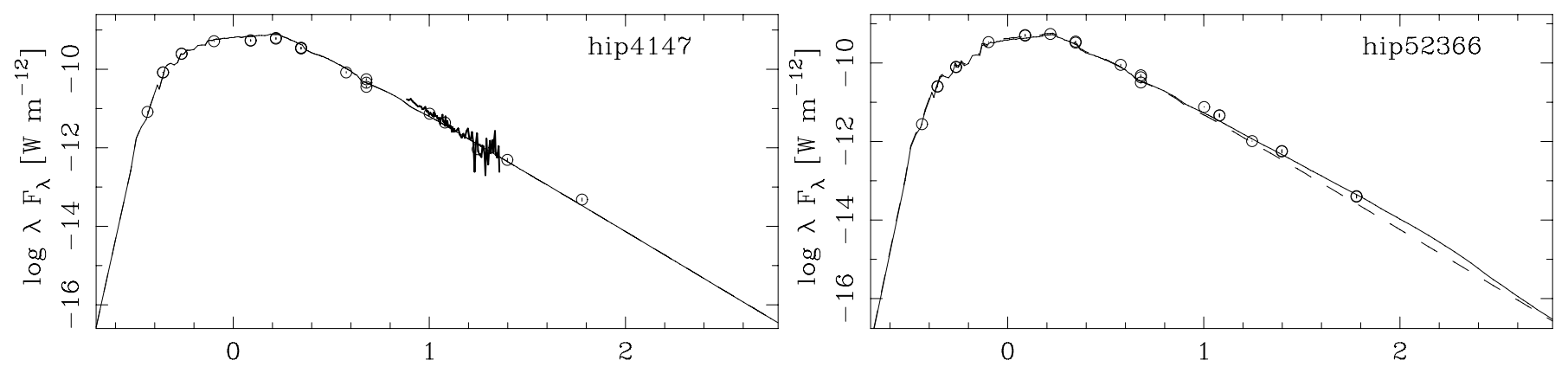

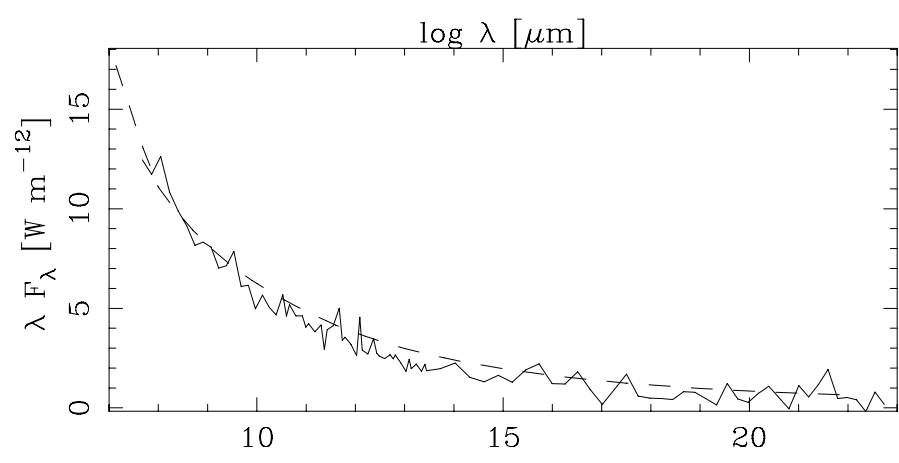

$\lambda[\mu \mathrm{m}]$

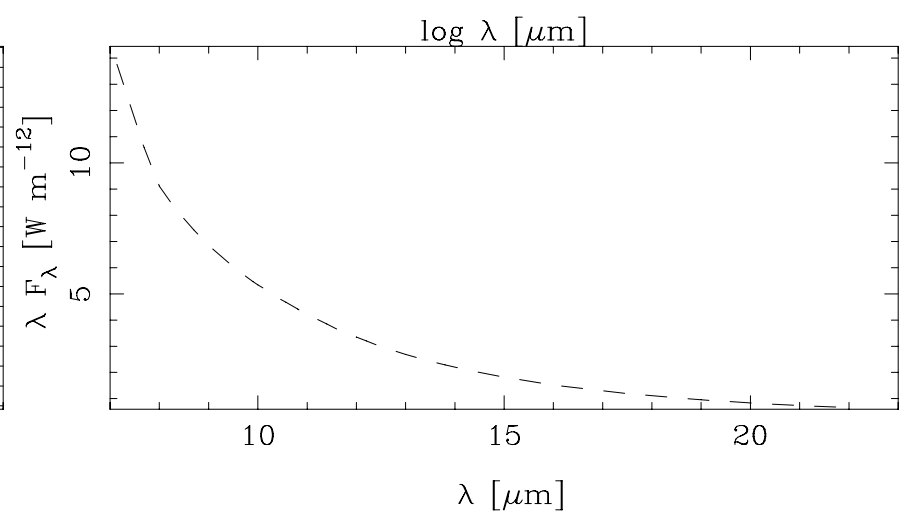

Fig. 2. Example fits to the SED (top panel) and IRAS LRS spectra (lower panel). In the top panel, the solid line indicates the best fit, the dashed line the model without mass loss. For HIP 4147, the best-fit model is the model without mass loss so that the two lines are over-plotted. The observed photometry is plotted by the circles, and error bars are also plotted, but are typically much smaller than the symbol size. In the lower panel, the best-fit model is indicated by the dashed line, and the LRS spectrum by the solid line. Sometimes no LRS spectrum is available, as for HIP 52366. The complete figure is available in Appendix A.
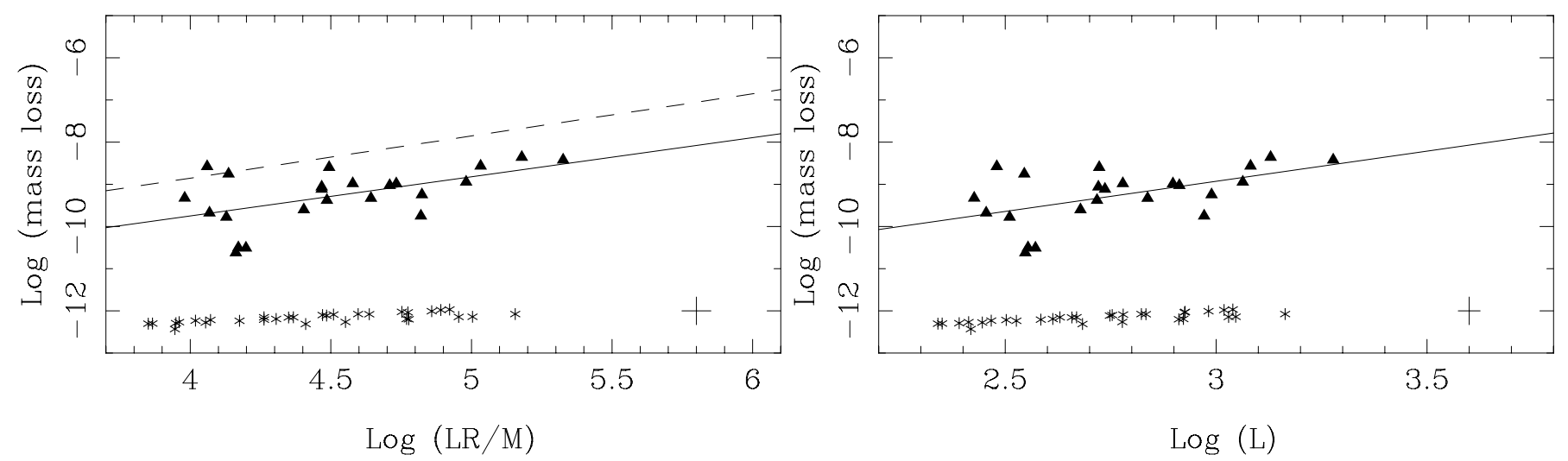

Fig. 3. Mass-loss rate plotted against $L R / M$ (for a mass of $1 M_{\odot}$ ) and $L$. Stars for which no mass loss could be detected (an optical depth of $10^{-5}$ ) are plotted as crosses. The solid lines indicate least squares fits to the data (see Table 3), while the dashed line represents Reimers law with $\eta=0.35$. The cross in the lower right corner indicates a typical error bar.

\section{Discussion}

\subsection{Mass loss}

Reimers law represents the Reimers (1975) mass-loss rate formula for red giants given by

$\dot{M}=\eta 4 \times 10^{-13}\left(\frac{L R}{M}\right)^{\gamma}\left(M_{\odot} \mathrm{yr}^{-1}\right)$

(with $\gamma=1$ and $\eta=1$, and $L, R$ and $M$ in solar units) which, interestingly, Kudritzki \& Reimers (1978) updated to $\dot{M}=(5.5 \pm$ $1) \times 10^{-13} \frac{L \cdot R}{M}\left(M_{\odot} \mathrm{yr}^{-1}\right)$, i.e. $\eta \approx 1.4$, by considering the mass loss in $\alpha$ Her, $\alpha$ Sco, and $\delta^{2}$ Lyr.
The left-hand panel of Fig. 3 shows the results of the current work, assuming a mass of $1.1 M_{\odot}$ for all stars. The PARAM tool ${ }^{3}$ (da Silva et al. 2006) was used to find that this is the typical mass of a star in the sample. An unweighted least squares fit gives $\log \dot{M}\left(M_{\odot} \mathrm{yr}^{-1}\right)=(0.9 \pm 0.3) \log (L R / M)+(-13.4 \pm 1.3)$. The slope is consistent with unity, but the coefficient $\left(3.7 \times 10^{-14}\right)$ is a factor of ten lower than in Reimers law. When $\gamma$ is fixed to unity, the coefficient becomes $1.8 \times 10^{-14}(\eta \approx 0.04)$ with an error of a factor of 3.4. The right-hand panel shows a fit of the massloss rate versus luminosity $\log \dot{M}\left(M_{\odot} \mathrm{yr}^{-1}\right)=(1.4 \pm 0.4) \log L+$ $(-13.2 \pm 1.2)$. Similar plots were made with the sample divided into $\mathrm{K}$ - and M-giants, according to Hipparcos variability type and effective temperature, but no clear trends were found. Fits

${ }^{3}$ http://stev.oapd.inaf.it/cgi-bin/param 
Table 3. Linear least squares fits to the mass-loss rates.

\begin{tabular}{|c|c|c|c|c|c|c|}
\hline Variable(s) & Zero point & Slope & Slope & $\chi^{2}$ & $\mathrm{BIC}$ & \\
\hline $\log R$ & $-13.76 \pm 1.30$ & $2.56 \pm 0.74$ & & 25.25 & 31.52 & \\
\hline $\log L R / M$ & $-13.43 \pm 1.26$ & $0.92 \pm 0.27$ & & 26.16 & 32.43 & \\
\hline $\log L$ & $-13.22 \pm 1.23$ & $1.42 \pm 0.44$ & & 26.88 & 33.16 & \\
\hline $\log T_{\text {eff }}$ & $52.17 \pm 20.3$ & $-17.2 \pm 5.7$ & & 28.10 & 34.73 & \\
\hline $\log R$ and $\log L$ & $-13.90 \pm 1.31$ & $5.1 \pm 3.4$ & $-1.5 \pm 2.1$ & 24.65 & 34.09 & \\
\hline $\log L R / M$ and $\log T_{\text {eff }}$ & $19.3 \pm 27.0$ & $0.65 \pm 0.35$ & $-8.8 \pm 7.3$ & 24.69 & 34.09 & \\
\hline $\log L$ & $-11.87 \pm 0.78$ & $0.99 \pm 0.27$ & & 50.34 & 58.08 & unweighted OLS \\
\hline $\log L R / M$ & $-11.83 \pm 0.78$ & $0.60 \pm 0.17$ & & 50.55 & 58.30 & unweighted OLS \\
\hline $\log R$ & $-11.16 \pm 0.87$ & $1.22 \pm 0.49$ & & 57.67 & 65.21 & unweighted OLS \\
\hline $\log L$ & $-12.00 \pm 0.94$ & $1.04 \pm 0.31$ & & & & BCES (OLS) \\
\hline $\log L R / M$ & $-11.90 \pm 0.92$ & $0.62 \pm 0.19$ & & & & BCES (OLS) \\
\hline
\end{tabular}

Notes. First entries are fits to the current sample (see Fig. 3). Last three entries includes literature mass-loss rates from modelling chromospheric lines in GC RGB stars (see Fig. 4).

against radius and effective temperature have also been made, and the results are compiled in Table 3. The best-fit relation is obtained when the mass-loss rate is fitted against (log) stellar radius.

Linear fits using two variables were also tested (Table 3), which resulted in lower $\chi^{2}$ but, according to the Bayesian information criterion (Schwarz 1978) where BIC $=\chi^{2}+(p+1) \ln (n)$ ( $p$ is the number of free parameters, and $n$ the number of data points), this is not significant as the BICs are larger.

These trends are in agreement with Catelan (2000), who in his appendix also presents several simple fitting formula that fit the data equally well. In the case of the fit against radius (his Eq. (A3)), he finds a slope of 3.2 (no error given), while we find a similar value of $2.6 \pm 0.7$. For a radius of $100 R_{\odot}$, Catelans' formula gives a mass-loss rate of $3.0 \times 10^{-9}$, while we find $2.2 \times$ $10^{-9} M_{\odot} \mathrm{yr}^{-1}$.

Among the 54 stars, 23 stars are found to have a significant infrared excess, which is interpreted as evidence for mass loss. The most luminous star with $L=1860 L_{\odot}$ is found to have mass loss, while none of the 5 stars with $L<262 L_{\odot}$ show evidence for mass loss. In the range $265<L<1500 L_{\odot}, 22$ stars out of 48 show mass loss, which supports the notion of episodic mass loss proposed by Origlia et al. (2007). They also find a shallower slope of $\gamma=0.4$, which the current data does not support. Catelan (2000) quotes $\gamma=1.4$.

The sample selection discussed in Sect. 2 involved imposing a lower limit of $(V-I)_{0}>1.5$, which could lead to a bias favouring mass-losing stars. To verify this, the $(V-I)$ colour predicted by the RT model of the mass-losing stars was compared to the colour of a model with the optical depth fixed to zero. Even for the star with the highest mass-loss rate, the model without mass loss is only bluer by $0.003 \mathrm{mag}$. This implies that the selection based on $(V-I)$ has no consequences for the statistics of the number and fraction of mass-losing stars in the sample.

Mass-loss rate estimates below the tip of the RGB exist mostly for stars in globular clusters (GCs), derived by both modelling the SEDs, as in the present paper, and modelling the chromospheric line profiles. Data were collected from the literature and are reproduced in Table 4. The first 24 entries are based on the modelling of the chromospheric line profiles, followed by the results of modelling the SEDs. For $\omega$ Cen, the values from McDonald et al. (2011) of the mass loss are preferred over those of McDonald et al. (2009) as they used Spitzer IRS spectra as additional constraints in the fitting.
All the SED modelling was performed in a similar way, also using DUSTY. However, in the McDonald et al. (2009, 2011, 2011) and Boyer et al. (2009) papers, DUSTY was run using a mode assuming radiatively-driven winds ("density type $=3$ "). The output velocity of DUSTY was then scaled as $\left(L / 10^{4}\right)^{(1 / 4)}(\Psi / 0.005)^{(1 / 2)}(\rho / 3)^{(-1 / 2)}$, and the mass-loss rate as $\left(L / 10^{4}\right)^{(3 / 4)}(\Psi / 0.005)^{(-1 / 2)}(\rho / 3)^{(1 / 2)}$. A dust-to-gas ratio of $\Psi=$ $0.005 \cdot 10^{[\mathrm{Fe} / \mathrm{H}]}$ was assumed in these models, which corresponds to very low outflow velocities: Boyer et al. (2009) mention values between 0.5 and $1.3 \mathrm{~km} \mathrm{~s}^{-1}$ for NGC 362, and McDonald et al. (2011) list values between 0.5 and $3.0 \mathrm{~km} \mathrm{~s}^{-1}$ for their sample in $\omega$ Cen.

Figure 4 is similar to Fig. 3 but with the literature data now added. There is excellent agreement with the mass-loss rates based on the modelling of the chromospheric activity, but an apparent discrepancy with the data that are also based on modelling the SEDs. This discrepancy is discussed in more detail in Sect. 5.2.

The results of unweighted least squares fits to the mass-loss rates derived in the present work and modelling of the chromospheric activity are reported in Table 3 and shown in Fig. 4. Adding the data from the chromospheric modelling leads to shallower, more accurately determined, slopes.

The use of unweighted least squares fits may be an oversimplification as both the abscissa and ordinate have (different) error bars. The error in the mass-loss rate is difficult to quantify. Following the discussion in Sect. 4, the internal fit error in the optical depth (typically 5\%), the error in the optical depth due to uncertainties in the stellar photosphere parameters (typically $50 \%$ ), and the uncertainty in the expansion velocity and dust-togas ratio when converting optical depth to mass-loss rates (assumed to be a factor of 2) are added in quadrature. The last error term dominates.

The error in the mass-loss rate derived by modelling the chromospheric lines is quoted to be a factor of two by Meszaros et al. (2009) and this is taken to be the error for all mass-loss rates derived by modelling the chromospheric lines. This means that the error bars along the ordinate are quite similar for all objects used in the fitting.

For the stars studied in the present work, the error in luminosity was derived by adding in quadrature the internal fit error (Table 2) and the error in the parallax (Table 1). The error in radius was calculated by taking the error in $L$ and a $70 \mathrm{~K}$ error in effective temperature. The error in mass was assumed to be 


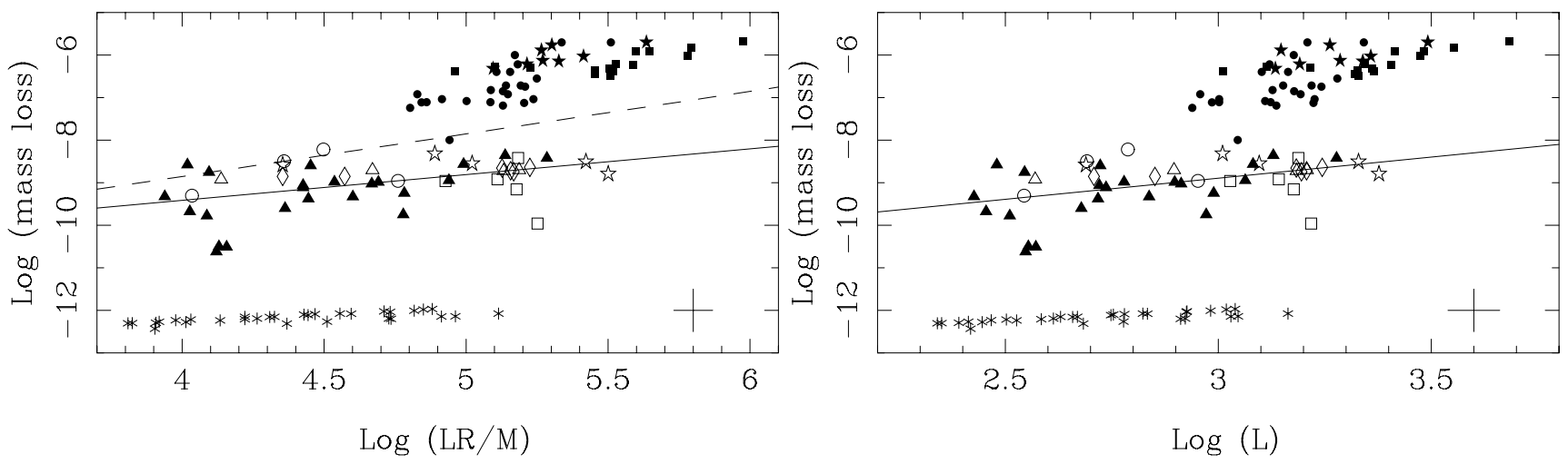

Fig. 4. As Fig. 3, but now for: this work (filled triangles, and the stars without excess as crosses), other data based on SED modelling: 47 Tuc (filled squares), $\omega$ Cen (filled circles), NGC 362 (filled stars), and modelling of chromospheric lines: M13 (open stars), M15 (open diamonds), M92 (open triangles), and $\omega$ Cen (open circles). The fit is to the present sample and the mass-loss rates from modelling the chromospheric lines, while the dashed line represents Reimers law with $\eta=0.35$. The cross in the lower right corner indicates a typical error bar.

$0.1 M_{\odot}$. For the stars in the present sample, typical error bars in $\log L$ and $\log L R / M$ are 0.026 , respectively 0.05 dex, and are shown in Fig. 3.

For the stars in the GCs it turns out that the luminosities quoted in the literature have been determined from $V$ and $K$ magnitudes and bolometric corrections. In addition there is the uncertainty in the distance modulus to the cluster. We assumed that an error bar of 0.15 in $M_{\text {bol }}$ was representative. The error in radius was calculated as before, while the error in mass was taken as $0.01 M_{\odot}$. The typical error bars in $\log L$ and $\log L R / M$ are 0.06 and 0.07 dex, respectively.

The "bivariate correlated errors and intrinsic scatter" (BCES) method was used (Akritas \& Bershady 1996) ${ }^{4}$, assuming that the errors along the ordinate and abscissa are uncorrelated. The results are very similar to those for the unweighted fitting, see Table 3, probably because the errors along the ordinate are similar for all data, and the errors along the abscissa are smaller than those in the ordinates. In what follows, the results from the BCES method are used.

\subsection{Are the winds dust driven?}

Figure 4 highlights an apparent discrepancy between the present work and the literature data also based on modelling the SEDs and that use the same numerical code.

The difference can be traced back to the way in which DUSTY is run, either with "density type $=1$ " and assuming a $r^{-2}$ density law, the mass-loss rate derived from the optical depth, the assumed dust-to-gas ratio and expansion velocity (as in the present paper), or, with "density type $=3$ " (which assumes the winds are driven by radiation pressure on dust), and then taking the DUSTY output for the mass-loss rate and expansion velocity, and applying scaling relations (see above) that take into account the luminosity and dust-to-gas ratio.

As a test, DUSTY models with "density type $=3$ " were also run for the present sample. The (scaled) mass-loss rates were then compared to the mass-loss rates from Table 2 scaling those to the predicted expansion velocities. Neglecting four outliers (with ratios above 100, which are among the five stars with the lowest mass-loss rates, hence optical depths that are probably particularly uncertain), the ratio of the mass-loss rates are between 18 and 60, with a median of 38 .

\footnotetext{
${ }^{4}$ http://www. astro.wisc. edu/ mab/archive/stats/stats. html
}

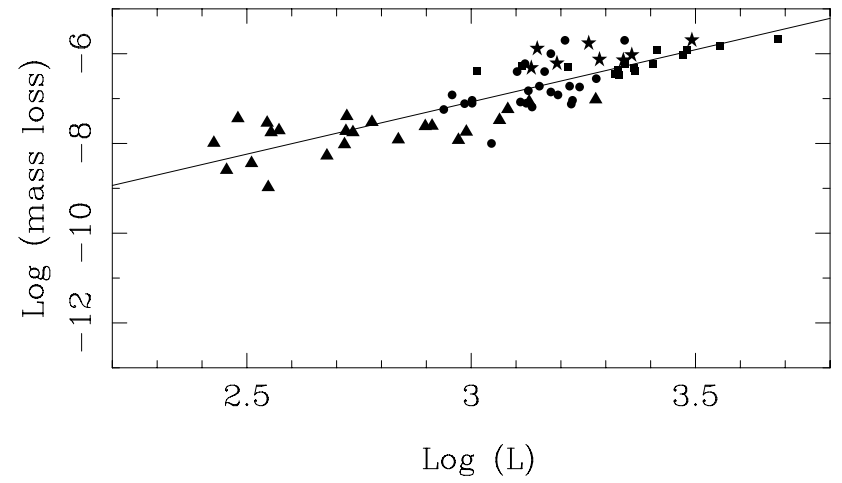

Fig. 5. As Fig. 3, with mass-loss rate plotted against luminosity, with mass-loss rates for the current sample calculated using DUSTY in "density type $=3$ ".

Figure 5 shows the new results together with the literature mass-loss rates from the SED modelling. The results appear now to be in much better agreement. We note that this suggests that there is a weak if any dependence of RGB mass loss on metallicity. The agreement between the mass-loss rates from the present modelling and the chromospheric line emission of the GC stars indicates the same.

There is however a good reason to be cautious when using DUSTY in the dust-driven wind mode and that is that the ratio of radiative forces to gravitation may be less than unity in some cases.

Equation (15) in Elitzur \& Ivezić (2001) derived this ratio to be $\Gamma=45.8 Q_{\star} \sigma_{22} L_{4} / M$, where $M$ is the stellar mass in solar units, $L_{4}$ the luminosity in units of $10^{4} L_{\odot}, Q_{\star}$ is the Planck average at the stellar temperature of the efficiency coefficient for radiation pressure (Eq. (4) in their paper), and $\sigma_{22}$ is the crosssection area per gas particle in units of $10^{-22} \mathrm{~cm}^{2}$ (see Eq. (5) in their paper). The latter quantity can be written as (with Eq. (86) in their paper) $\sigma_{22}=125.4 \Psi /(\rho a)$, with $\Psi$ the dust-to-gas ratio, $\rho$ the grain specific density in $\mathrm{g} \mathrm{cm}^{-3}$, and $a$ the grain size in micron.

In the optically thin case, $Q_{\star}$ can be taken as the sum of absorption and scattering coefficients at the wavelength where the stellar photosphere peaks. For the typical effective temperatures considered here, this is at about 1.6 micron, and $Q_{\star} \sim 2.2$ (for the iron dust properties discussed in Sect. 3). With $\rho=5.1$, $a=0.15$ and $\Psi=0.005, \sigma_{22}$ equals 0.82 , and then, assuming 
Table 5. Comparison of angular diameters.

\begin{tabular}{lcccc}
\hline \hline \multirow{2}{*}{ HIP } & & \multicolumn{2}{c}{$\theta$ and error (mas) } & \\
& Bordé et al. (2002) & Mozurkewich et al. (2003) & Richichi et al. (2009) & This paper (Table 2) \\
\hline 4147 & $3.51 \pm 0.037$ & $3.459 \pm 0.006$ & $3.85 \pm 0.15$ \\
12107 & $3.11 \pm 0.032$ & & $3.47 \pm 0.15$ \\
32173 & $2.73 \pm 0.029$ & & $3.03 \pm 0.13$ \\
37300 & $3.28 \pm 0.038$ & & $3.43 \pm 0.13$ \\
41822 & $2.88 \pm 0.031$ & & $2.58 \pm 0.11$ \\
44857 & $2.67 \pm 0.035$ & & $3.17 \pm 0.13$ \\
46750 & $4.12 \pm 0.046$ & & & $4.59 \pm 0.18$ \\
49005 & $2.18 \pm 0.023$ & & & $2.15 \pm 0.08$ \\
53907 & $3.87 \pm 0.041$ & & & $3.95 \pm 0.16$ \\
55687 & $3.57 \pm 0.038$ & & & $3.31 \pm 0.12$ \\
56127 & $3.03 \pm 0.034$ & & & $3.41 \pm 0.16$ \\
56211 & & & $6.85 \pm 0.27$ \\
60122 & $3.34 \pm 0.038$ & & & $3.60 \pm 0.14$ \\
64607 & $2.29 \pm 0.026$ & & & $2.26 \pm 0.11$ \\
67665 & $5.35 \pm 0.059$ & & & $5.64 \pm 0.24$ \\
77661 & $3.40 \pm 0.041$ & & & $3.35 \pm 0.12$ \\
82073 & $2.84 \pm 0.029$ & & & $2.80 \pm 0.11$ \\
84835 & $2.60 \pm 0.027$ & & & $2.67 \pm 0.11$ \\
87833 & & & & $10.55 \pm 0.38$ \\
88122 & $2.42 \pm 0.027$ & $9.860 \pm 0.128$ & & $2.07 \pm 0.08$ \\
106140 & & & & $4.89 \pm 0.19$ \\
112716 & $5.12 \pm 0.053$ & $4.521 \pm 0.047$ & & $4.98 \pm 0.19$ \\
\hline
\end{tabular}

$M=1, \Gamma$ becomes 8.3 for $L_{4}=0.1$. In this case, $\Gamma>1$ and so the condition to drive the outflow by radiation pressure on dust is met. For the lowest luminosities in which we detect an excess, $L$ is $\sim 260 L_{\odot}$ and so $\Gamma \sim 2$.

In the case of the GC stars from the literature, the majority of luminosities are above $L_{4}=1$ (see Fig. 4) but these authors assumed the dust-to-gas ratio to scale with metallicity, which results in very low values in the range $1 / 1200$ to $1 / 5000$ in the case of $\omega$ Cen (McDonald et al. 2011), and again, $\Gamma$ would be close to or even below unity.

The largest uncertainty in predicting $\Gamma$ may be in the calculation of the dust properties. As mentioned in the introduction, there are indications that metallic iron may be abundant and the fact that the infrared excess is featureless is one of them.

A value of $Q_{\star}=2.2$ is much larger than that found for standard silicates or carbon dust (Table 1 in Elitzur \& Ivezić 2001, lists 0.1 and 0.6 , respectively, for $0.1 \mu \mathrm{m}$ sized grains). The adopted grain size is of importance. In the standard case, $Q / a=14.6 \mu \mathrm{m}^{-1}$, but this value decreases (and hence $\Gamma$ decreases) to 9.4 and 6.1 for $a=0.1$ and $0.05 \mu \mathrm{m}$, respectively. This effect can also lead to a larger $\Gamma: Q / a$ peaks at $21.6 \mu \mathrm{m}^{-1}$ for $a=0.23 \mu \mathrm{m}$. The influence on grain size is largely due to the scattering contribution and this implies that even the assumption of isotropic scattering can play a role. The assumption on the grain morphology is also important. If the DHS is used with a smaller maximum fraction of vacuum $Q /(a \rho)$ is also reduced: by a factor of 2 in the case of $f_{\max }=0.4$ and a factor of 3.2 in the case of $f_{\max }=0.0$ (i.e. compact spherical grains).

Elitzur \& Ivezić (2001) derive in a similar way a more stringent constraint on the minimal mass-loss rate (their Eq. (69)), $\dot{M} \gtrsim 3 \times 10^{-9} \frac{M}{Q_{\star} \sigma_{22}^{2} L_{4} T_{\mathrm{k} 3}^{0.5}}$, which corresponds to $2 \times 10^{-8} M_{\odot} \mathrm{yr}^{-1}$ for the above values for $M, Q_{\star}$, and $\sigma_{22}, L_{4}=0.1$, and $T_{\mathrm{k} 3}=1$, which is the kinetic temperature at the inner radius in units of $1000 \mathrm{~K}$. On the basis of this consideration. all mass-loss rates in Table 2 are below this critical value.

\subsection{Angular diameters}

Angular diameters are a prediction of the radiative transfer modelling through the fitting of the luminosity and the effective temperature. The values are listed in Table 2. The (limb darkened) angular diameters of some stars determined from interferometry and the predicted values from the SED modelling are compared in Table 5.

The largest overlap is that with Bordé et al. (2002), which is based on the results by Cohen et al. (1999). The overall agreement is good, leading to ( $\theta_{\text {Borde et al }}-$ $\left.\theta_{\text {present work }}\right) /\left(\sqrt{\sigma_{\text {Borde et al }}^{2}+\sigma_{\text {present work }}^{2}}\right)$ of $-1.0 \pm 1.7$. There are 2 stars where the difference is more than $3 \sigma$, HIP 44857 and HIP 88122. In the latter case, this is due to the different adopted effective temperatures, $4000 \mathrm{~K}$ in the present paper, and $3690 \mathrm{~K}$ in Bordé et al., which is the largest difference in temperature among the 19 stars in common.

The overlap with Mozurkewich et al. (2003) occurs for three sources, for which the agreement is satisfactory. These authors obtained their data mostly at 550 and $800 \mathrm{~nm}$ and therefore the limb-darkening correction is relatively large. The main difference is found for HIP 87833, for which Mozurkewich et al. quote angular diameters based on the infrared flux method of 10.450 (Bell \& Gustafsson 1989) and 10.244 mas (Blackwell et al. 1990), which are in excellent agreement with the present work. Finally, two stars overlap with Richichi et al. (2009), and the agreement is satisfactory.

\section{Summary and conclusions}

We have selected a sample of 54 nearby RGB stars, and constructed and modelled their SEDs with a dust radiative transfer code. In about half of the stars, the SEDs were statistically better fitted when we included a (featureless) dust component. The lowest luminosity is which a significant excess was found is $267 L_{\odot}$ (HIP 64607), which is lower than for any other star (as far as I am aware). 
Table 6. Mass lost on the RGB for a $1.6 M_{\odot}$ star.

\begin{tabular}{lccc}
\hline \hline Relationship & $\begin{array}{c}\Delta M \\
\left(M_{\odot}\right)\end{array}$ & $\begin{array}{c}\dot{M}\left(\text { at } L=1000 L_{\odot}\right) \\
10^{-9} M_{\odot} \mathrm{yr}^{-1}\end{array}$ & Remark \\
\hline $1.0 \log (L R / M)-12.853$ & 0.082 & 8.2 & Reimers law $\eta=0.35$ \\
$0.6 \log (L R / M)-11.9$ & 0.008 & 0.9 & \\
$0.8 \log (L R / M)-11.9$ & 0.077 & 8.3 & \\
$0.4 \log (L R / M)-11.9$ & 0.001 & 0.10 & \\
$0.6 \log (L R / M)-11.0$ & 0.067 & 7.3 & \\
$0.6 \log (L R / M)-12.8$ & 0.001 & 0.11 & \\
$1.0 \log (L)-12.0$ & 0.009 & 1.0 & \\
$1.3 \log (L)-12.0$ & 0.073 & 7.9 & \\
$0.7 \log (L)-12.0$ & 0.001 & 0.12 & \\
$1.0 \log (L)-11.1$ & 0.073 & 7.9 & \\
$1.0 \log (L)-12.9$ & 0.001 & 0.12 & \\
\hline
\end{tabular}

The results are compared with mass-loss rates estimated for RGB stars in GCs, based on modelling chromospheric line emission and the dust excess. There is good agreement with the former sample, and fits of the mass-loss rate against luminosity and $(L R / M)$ (Reimers law) are presented by combining the present work with the mass-loss rates from the chromospheric modelling. The derived slope is shallower then in Reimers law and with a larger constant. The comparison with the literature values based on modelling the dust excess has led to an interesting observation. This is that there is a significant difference among (and dependence on luminosity for) the mass-loss rates derived when running the DUSTY code in "density type $=1$ " and assuming a $r^{-2}$ density law, and then deriving the mass-loss rate from the optical depth, an assumed dust-to-gas ratio, and expansion velocity (as in the present paper), or, with "density type $=3$ " (that assumes the winds are driven by radiation pressure on dust), and then taking the DUSTY output for the mass-loss rate and expansion velocity, and applying scaling relations that take into account the luminosity and dust-to-gas ratio (as done in the literature). The origin of the difference is unclear, but it turns out that the ratio of radiative forces to gravitation $(\Gamma)$ could be smaller than unity under certain conditions for the RGB stars we consider, for low luminosity and/or low dust-to-gas ratios (as assumed in the GC stars). In addition, the details of the dust that forms (iron dust has been assumed in the nearby RGB stars, as it is found to be the dominant dust species in the GC sample), and the typical grain size and morphology could play a crucial role in whether $\Gamma$ is larger than unity in any given star. This might explain why only 22 out of $48 \mathrm{RGB}$ stars with $265<L<1500 L_{\odot}$ have an infrared excess. The condition $\Gamma>1$ may only be fulfilled for $50 \%$ of the time (or, instantaneously, in $50 \%$ of the stars) in this luminosity range when certain conditions are met. What these conditions or triggers are remains unclear: they could be related to either pulsation or convection or be more indirect as for binarity or magnetic fields. Determining the outflow velocity of the wind for the stars that show an excess would be helpful because this would not only remove the assumption of a constant velocity of $10 \mathrm{~km} \mathrm{~s}^{-1}$ for all objects, but also test the predictions of the dust-driven wind theory.

To investigate the implications of the mass-loss rate formula derived here, we compared the predicted mass loss on the RGB to the recent determination from asteroseismology for the cluster NGC 6791 (Miglio et al. 2012), who derive a mass loss of $\Delta M=0.09 \pm 0.03$ (random) \pm 0.04 (systematic) $M_{\odot}$. Evolutionary tracks were used from the same dataset (Bertelli et al. 2008 $)$ and with the same composition $(Z=0.04$, $Y=0.33$ ) as used by Miglio et al. For a star with initial mass

\footnotetext{
5 http://stev.oapd.inaf.it/YZVAR/
}

1.6 $M_{\odot}$ (Miglio et al. determine the mass on the RGB to be $1.61 M_{\odot}$ ), the mass lost on the RGB was calculated when the luminosity was above $250 L_{\odot}$ (the effect of including the entire RGB is negligible) for different mass-loss recipes (see Table 6). The first entry is for Reimers law with a scaling of $\eta=0.35$, which gives a total mass lost on the RGB close to the observed value. The following rows provide the predictions from the bestfit relations derived in this paper with $L$ and $L R / M$ as variables and varying the slope and zero point by their respective $1 \sigma$ error bars. Both type of relations can equally well result in the observed mass lost, for a slope and/or zero point slightly larger than the best-fit value. As for the scaling of the Reimers law, the following relation would fit the observed mass loss of $0.09 \pm 0.05 M_{\odot}$ equally well: $\dot{M}=\eta_{1} 1.25 \times 10^{-12}\left(\frac{L R}{M}\right)^{0.6}$ with $\eta_{1}=11 \pm 6$, and $\dot{M}=\eta_{2} 1.00 \times 10^{-12}(L)^{1.0}$ with $\eta_{1}=10 \pm 5$. That the scaling factors are larger than unity would suggest, in the framework of the dust modelling, that the expansion velocities and/or dust-to-gas ratios (or even the dust opacities) different from those assumed.

The table also lists the predicted mass-loss rate at a luminosity of $1000 L_{\odot}$, and for the models that predict the observed total mass loss, this mass-loss rate is about $8 \times 10^{-9} M_{\odot} \mathrm{yr}^{-1}$. Comparing this to Figs. 3 and 5 gives independent evidence that the mass-loss rates in Table 4 based on DUSTY using "density type $=3$ " appear to be too large by an order of magnitude.

Acknowledgements. M.G. would like to thank Dr. Iain McDonald for the very fruitful discussion regarding the input to DUSTY and commenting on a draft version of the paper, and the referee for suggesting additional checks, and pointing out the PARAM website. This research has made use of the SIMBAD database, operated at CDS, Strasbourg, France.

\section{References}

Akritas, M. G., \& Bershady, M. A. 1996, ApJ, 470, 706

Arenou, F., Grenon, M., \& Gomez, A. 1992, A\&A, 258, 104

Begemann, B., Dorschner, J., \& Henning, Th., et al. 1997, ApJ, 476, 199

Beichman, C. A., Neugebauer, G., Habing, H. J., Clegg, P. E., \& Chester, T. J. 1985, in IRAS Explamatory Supplement (Pasadena: JPL)

Bell, R. A., \& Gustafsson, B. 1989, MNRAS, 236, 653

Bertelli, G., Girardi, L., Marigo, P., \& Nasi, E. 2008, A\&A, 484, 815

Blackwell, D. E., Petford, A. D., Arribas, S., Haddock, D. J., \& Shelby, M. J. 1990, A\&A, 232, 396

Bordé, P., Coudé du Foresto, V., Chagnon, G., \& Perrin, G. 2002, A\&A, 393, 183

Boyer, M. L., McDonald, I., van Loon, J. Th., et al. 2009, ApJ, 705, 746

Boyer, M. L., van Loon, J. Th., McDonald, I., et al. 2010, ApJ, 711, L99

Caloi, V., \& d'Antona, F. 2008, ApJ, 673, 847

Catelan, M. 2000, ApJ, 531, 826

Cenarro, A. J., Peletier, R. F., Sánchez-Blázquez, P., et al. 2007, MNRAS, 374, 664

Clement, C. M., Muzzin, A., Dufton, Q., et al. 2001, AJ, 122, 2587

Cohen, M., Walker, R. G., Carter, B., et al. 1999, AJ, 117, 1864 
M. A. T. Groenewegen: Infrared excess around nearby red giant branch stars and Reimers law

da Silva, L., Girardi, L, Pasquini, L., et al. 2006, A\&A, 458, 609

Drimmel, R., Cabrera-Lavers, A., \& López-Corredoira, M. 2003, A\&A, 409, 205

Dumm, T., \& Schild, H. 1998, NewA, 3, 137

Elitzur, M., \& Ivezić, Ž. 2001, MNRAS, 327, 403

ESA 1997, The HipPARcos Catalogue, ESA SP-1200 (viZier catalog I/239)

Fernández-Villacañas, J. L., Rego, M., \& Cornide, M. 1990, AJ, 99, 1961

Gezari, D. Y., Pitts, P. S., \& Schmitz, M. 1999, in Catalog of Infrared Observations, edn. 5 (viZier catalog II/225)

Groenewegen, M. A. T. 2008, A\&A, 488, 935

Groenewegen, M. A. T., Sloan, G. C., Soszyński, I., \& Petersen, E. A. 2009, A\&A, 506, 1277

Gustafsson, B., Edvardsson, B., Eriksson, K., et al. 2008, A\&A, 486, 951

Habing, H. J., \& Olofsson, H. 2003, in Asymptotic Giant Branch Stars (New York: Springer-Verlag)

Ishihara, D., Onaka, T., Kataza, H., et al. 2010, A\&A, 514, A1 (viZier catalog II/297)

Ivezić, Ž., Nenkova, M., \& Elitzur, M. 1999, DUSTY user manual, University of Kentucky internal report

Koen, C., \& Eyer, L. 2002, MNRAS, 331, 45

Koen, C., \& Laney, D. 2000, MNRAS, 311, 636

Kudritzki, R., \& Reimers, D. 1978, A\&A, 70, 227

Lebzelter, T., \& Wood, P. R. 2005, A\&A, 441, 1117

Marshall, D. J., Robin, A. C., Reyle, C., Schultheis, M., \& Picaud, S. 2006, A\&A, 453, 635

Massarotti, A., Latham, D. W., Stefanek, R. P., et al. 2008, AJ, 135, 209

Mauas, P. J. D., Cacciari, C., \& Pasquini, L. 2006, A\&A, 454, 609

Mermilliod, J. C. 1991, in Catalogue of Homogeneous Means in the UBV System (viZier catalog II/168)

McDonald, I., van Loon, J. Th., Decin, L., et al. 2009, MNRAS, 394, 831

McDonald, I., Sloan, G. C., Zijlstra, A. A., et al. 2010, ApJ, 717, L92
McDonald, I., Boyer, M. L., van Loon, J. Th., \& Zijlstra, A. A. 2011a, ApJ, 730, 71

McDonald, I., van Loon, J. Th., Sloan, G. C., et al. 2011b, MNRAS, 417, 20

McWilliam, A. 1990, ApJS, 74, 1075

Mészáros, Sz., Avrett, E. H., \& Dupree, A. K. 2009, AJ, 138, 615

Miglio, A., Brogaard, K., Stello, D., et al. 2012, MNRAS, 419, 2077

Min, M., Hovenier, J. W., \& de Koter, A. 2005, A\&A, 432, 909

Momany, Y., Saviane, I., Smette, A., et al. 2012, A\&A, 537, A2

Moshir, M., Kopan, G., Conrow, T., et al. 1989, in Explanatory supplement to the IRAS Faint Source Survey (Pasadena: JPL)

Mozurkewich, D., Armstron, J. T., Hindsley, R. B., et al. 2003, AJ, 126, 2502

Niyogi, S. G., Speck, A., \& Onaka, T. 2011, ApJ, 733, 93

Olnon, F. M., Raimond, E., Neugebauer, G., et al. 1986, A\&AS, 65, 607

Origlia, L., Rood, R. T., Fabbri, S., et al. 2007, ApJ, 667, L85

Origlia, L., Rood, R. T., Fabbri, S., et al. 2010, ApJ, 718, 522

Planck Collaboration 2011, A\&A, 537, A7

Pollack, J. B., Hollenbach, D., Beckwith, S., et al. 1994, ApJ, 421, 615

Reimers, D. 1975, MSRSL, 8, 369

Richichi, A., Percheron, I., \& Davis, J. 2009, MNRAS, 399, 399

Schwarz, G. 1978, Ann. Stat., 6, 461

Tsuji, T. 2000, ApJ, 538, 801

van Leeuwen, F. 2007, in HIPPARcos, the new reduction of the raw data (Dordrecht: Springer)

Verhoelst, T., Decin, L., van Malderen, R., et al. 2006, A\&A, 447, 311

Verhoelst, T., Van der Zypen, N., Hony, S., et al. 2009, A\&A, 498, 127

Vieytes, M., Mauas, P., Cacciari, C., Origlia, L., \& Pancino, E. 2011, A\&A, 526 A4

Volk, K., \& Cohen, M. 1989, AJ, 98, 931

Yamamura, I., Makiuti, S., Ikeda, N., et al. 2010, ISAS/JAXA (viZier catalog II/298)

Pages 12 to 21 are available in the electronic edition of the journal at http://www . aanda. org 
Table 4. GC data for mass-losing stars.

\begin{tabular}{|c|c|c|c|c|c|c|}
\hline Cluster & Identifier & $\begin{array}{c}\text { Mass-loss rate } \\
\left(M_{\odot} \mathrm{yr}^{-1}\right)\end{array}$ & {$[\mathrm{Fe} / \mathrm{H}]$} & $\begin{array}{l}T_{\text {eff }} \\
(\mathrm{K})\end{array}$ & $\begin{array}{c}\log L \\
\left(L_{\odot}\right)\end{array}$ & Reference \\
\hline M13 & L72 & $2.8 \times 10^{-09}$ & -1.54 & 4180. & 3.096 & Meszaros et al. (2009) \\
\hline M13 & L96 & $4.8 \times 10^{-09}$ & -1.54 & 4190. & 3.010 & \\
\hline M13 & L592 & $2.6 \times 10^{-09}$ & -1.54 & 4460. & 2.689 & \\
\hline M13 & L954 & $3.1 \times 10^{-09}$ & -1.54 & 3940. & 3.329 & \\
\hline M13 & L973 & $1.6 \times 10^{-09}$ & -1.54 & 3910. & 3.377 & \\
\hline M15 & K87 & $1.4 \times 10^{-09}$ & -2.26 & 4610. & 2.708 & \\
\hline M15 & K341 & $2.2 \times 10^{-09}$ & -2.26 & 4300. & 3.183 & \\
\hline M15 & K421 & $1.9 \times 10^{-09}$ & -2.26 & 4330. & 3.207 & \\
\hline M15 & K479 & $2.3 \times 10^{-09}$ & -2.26 & 4270. & 3.244 & \\
\hline M15 & K757 & $1.8 \times 10^{-09}$ & -2.26 & 4190. & 3.195 & \\
\hline M15 & K969 & $1.4 \times 10^{-09}$ & -2.26 & 4590. & 2.851 & \\
\hline M92 & VII18 & $2.0 \times 10^{-09}$ & -2.28 & 4190. & 3.208 & \\
\hline M92 & X49 & $1.9 \times 10^{-09}$ & -2.28 & 4280. & 3.184 & \\
\hline M92 & XII8 & $2.0 \times 10^{-09}$ & -2.28 & 4430. & 2.896 & \\
\hline M92 & XII34 & $1.2 \times 10^{-09}$ & -2.28 & 4660. & 2.570 & \\
\hline NGC 2808 & 37872 & $1.1 \times 10^{-09}$ & -1.14 & 4015. & 3.028 & Mauas et al. (2006) \\
\hline NGC 2808 & 47606 & $1.1 \times 10^{-10}$ & -1.14 & 3839. & 3.218 & \\
\hline NGC 2808 & 48889 & $3.8 \times 10^{-09}$ & -1.14 & 3943. & 3.188 & \\
\hline NGC 2808 & 51454 & $0.7 \times 10^{-09}$ & -1.14 & 3893. & 3.177 & \\
\hline NGC 2808 & 51499 & $1.2 \times 10^{-09}$ & -1.14 & 3960. & 3.142 & \\
\hline$\omega \mathrm{Cen}$ & ROA159 & $1.1 \times 10^{-09}$ & -1.72 & 4200. & 2.952 & Vieytes et al. (2011) \\
\hline$\omega$ Cen & ROA256 & $6.0 \times 10^{-09}$ & -1.71 & 4300. & 2.788 & \\
\hline$\omega$ Cen & ROA238 & $3.2 \times 10^{-09}$ & -1.80 & 4200. & 2.692 & \\
\hline$\omega$ Cen & ROA523 & $5.0 \times 10^{-10}$ & -0.65 & 4200. & 2.544 & \\
\hline 47 Tuc & V1 & $2.1 \times 10^{-06}$ & -0.7 & 3623. & 3.683 & McDonald et al. (2011) \\
\hline 47 Tuc & V8 & $1.5 \times 10^{-06}$ & -0.7 & 3578. & 3.554 & \\
\hline $47 \mathrm{Tuc}$ & V2 & $1.2 \times 10^{-06}$ & -0.7 & 3738. & 3.482 & \\
\hline $47 \mathrm{Tuc}$ & V3 & $9.4 \times 10^{-07}$ & -0.7 & 3153. & 3.473 & \\
\hline 47 Tuc & V4 & $1.2 \times 10^{-06}$ & -0.7 & 3521. & 3.415 & \\
\hline 47 Tuc & V26 & $5.9 \times 10^{-07}$ & -0.7 & 3500. & 3.405 & \\
\hline 47 Tuc & LW10 & $4.2 \times 10^{-07}$ & -0.7 & 3543. & 3.366 & \\
\hline 47 Tuc & V21 & $4.9 \times 10^{-07}$ & -0.7 & 3575. & 3.362 & \\
\hline 47 Tuc & LW9 & $6.0 \times 10^{-07}$ & -0.7 & 3374. & 3.343 & \\
\hline $47 \mathrm{Tuc}$ & V27 & $3.3 \times 10^{-07}$ & -0.7 & 3374. & 3.330 & \\
\hline 47 Tuc & L1424 & $4.4 \times 10^{-07}$ & -0.7 & 3565. & 3.327 & \\
\hline 47 Tuc & A19 & $3.6 \times 10^{-07}$ & -0.7 & 3526. & 3.321 & \\
\hline $47 \mathrm{Tuc}$ & $\mathrm{x} 03$ & $5.1 \times 10^{-07}$ & -0.7 & 3816. & 3.215 & \\
\hline 47 Tuc & V18 & $5.3 \times 10^{-07}$ & -0.7 & 3692. & 3.113 & \\
\hline 47 Tuc & V13 & $4.1 \times 10^{-07}$ & -0.7 & 3657. & 3.012 & \\
\hline$\omega$ Cen & 52111 & $5.7 \times 10^{-08}$ & -1.62 & 3975. & 2.939 & McDonald et al. (2009) \\
\hline$\omega$ Cen & 25062 & $1.2 \times 10^{-07}$ & -1.83 & 4150. & 3.193 & \\
\hline$\omega$ Cen & 43351 & $9.1 \times 10^{-08}$ & -1.62 & 3895. & 3.002 & \\
\hline$\omega$ Cen & 36036 & $7.8 \times 10^{-08}$ & -2.05 & 3944. & 3.123 & \\
\hline$\omega \mathrm{Cen}$ & 42205 & $7.7 \times 10^{-08}$ & -1.62 & 4110. & 2.985 & \\
\hline$\omega$ Cen & 26025 & $7.5 \times 10^{-08}$ & -1.68 & 4088. & 3.223 & \\
\hline$\omega$ Cen & 45232 & $2.8 \times 10^{-07}$ & -1.62 & 4276. & 3.279 & \\
\hline$\omega$ Cen & 49123 & $1.9 \times 10^{-07}$ & -1.62 & 3895. & 3.152 & \\
\hline$\omega$ Cen & 48060 & $1.9 \times 10^{-07}$ & -1.97 & 4117. & 3.219 & \\
\hline$\omega$ Cen & 56087 & $1.8 \times 10^{-07}$ & -1.92 & 4209. & 3.242 & \\
\hline$\omega \mathrm{Cen}$ & 41455 & $1.5 \times 10^{-07}$ & -1.29 & 3966. & 3.127 & \\
\hline$\omega$ Cen & 32138 & $1.4 \times 10^{-07}$ & -1.87 & 4124. & 3.178 & \\
\hline$\omega$ Cen & 37110 & $1.2 \times 10^{-07}$ & -1.62 & 3981. & 2.957 & \\
\hline$\omega$ Cen & 47153 & $1.0 \times 10^{-08}$ & -1.62 & 4070. & 3.045 & \\
\hline$\omega$ Cen & 48150 & $9.2 \times 10^{-08}$ & -1.62 & 3956. & 3.226 & \\
\hline$\omega$ Cen & 42302 & $8.3 \times 10^{-08}$ & -1.62 & 4245. & 3.110 & \\
\hline$\omega$ Cen & 39165 & $7.8 \times 10^{-08}$ & -1.62 & 4144. & 3.002 & \\
\hline$\omega$ Cen & 39105 & $6.5 \times 10^{-08}$ & -0.85 & 3833. & 3.136 & \\
\hline$\omega$ Cen & 33062 & $2.0 \times 10^{-06}$ & -1.08 & 3534. & 3.342 & McDonald et al. (2011) \\
\hline$\omega$ Cen & 44262 & $2.0 \times 10^{-06}$ & -0.8 & 3427. & 3.209 & \\
\hline$\omega$ Cen & 44277 & $1.0 \times 10^{-06}$ & -1.37 & 3921. & 3.177 & \\
\hline$\omega$ Cen & 55114 & $4.0 \times 10^{-07}$ & -1.45 & 3906. & 3.164 & \\
\hline$\omega$ Cen & 35250 & $6.0 \times 10^{-07}$ & -1.06 & 3513. & 3.120 & \\
\hline$\omega$ Cen & 42044 & $4.0 \times 10^{-07}$ & -1.37 & 3708. & 3.102 & \\
\hline NGC 362 & $\mathrm{~s} 02$ & $1.7 \times 10^{-06}$ & -1.16 & 3907. & 3.262 & Boyer et al. (2009) \\
\hline NGC 362 & s03 & $7.1 \times 10^{-07}$ & -1.16 & 4339. & 3.339 & \\
\hline NGC 362 & s04 & $6.1 \times 10^{-07}$ & -1.16 & 3823. & 3.191 & \\
\hline NGC 362 & s05 & $9.3 \times 10^{-07}$ & -1.16 & 4058. & 3.358 & \\
\hline NGC 362 & s06 & $2.0 \times 10^{-06}$ & -1.16 & 3962. & 3.492 & \\
\hline NGC 362 & s07 & $1.3 \times 10^{-06}$ & -1.16 & 3343. & 3.147 & \\
\hline NGC 362 & s09 & $7.4 \times 10^{-07}$ & -1.16 & 4226. & 3.286 & \\
\hline NGC 362 & s10 & $4.8 \times 10^{-07}$ & -1.16 & 3975. & 3.134 & \\
\hline
\end{tabular}


M. A. T. Groenewegen: Infrared excess around nearby red giant branch stars and Reimers law

\section{Appendix A: Fits to the SEDs}

All fits are shown here.
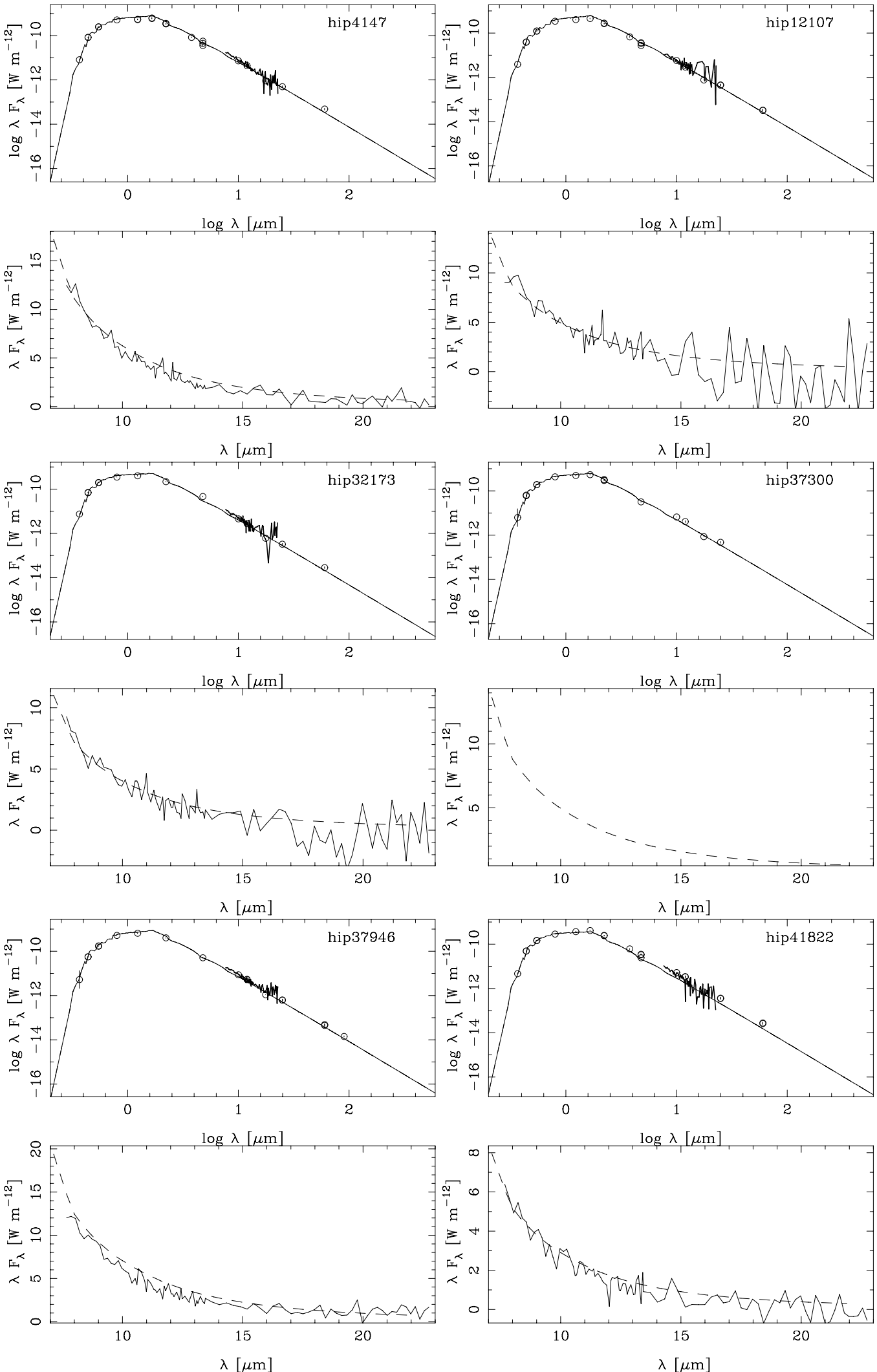

Fig. A.1. Fits to the SED (top panel) and IRAS LRS spectra (lower panel). In the top panel, the solid line indicates the best fit, the dashed line the model without mass loss (in many cases the two models overlap and are indistinguishable). The observed photometry is inidicated by the circles, and error bars are also plotted, but typically are much smaller than the symbol size. In the lower panel, the best-fit model is indicated by the dashed line, and the LRS spectrum by the solid line. Sometimes no LRS spectrum was available. 
A\&A 540, A32 (2012)
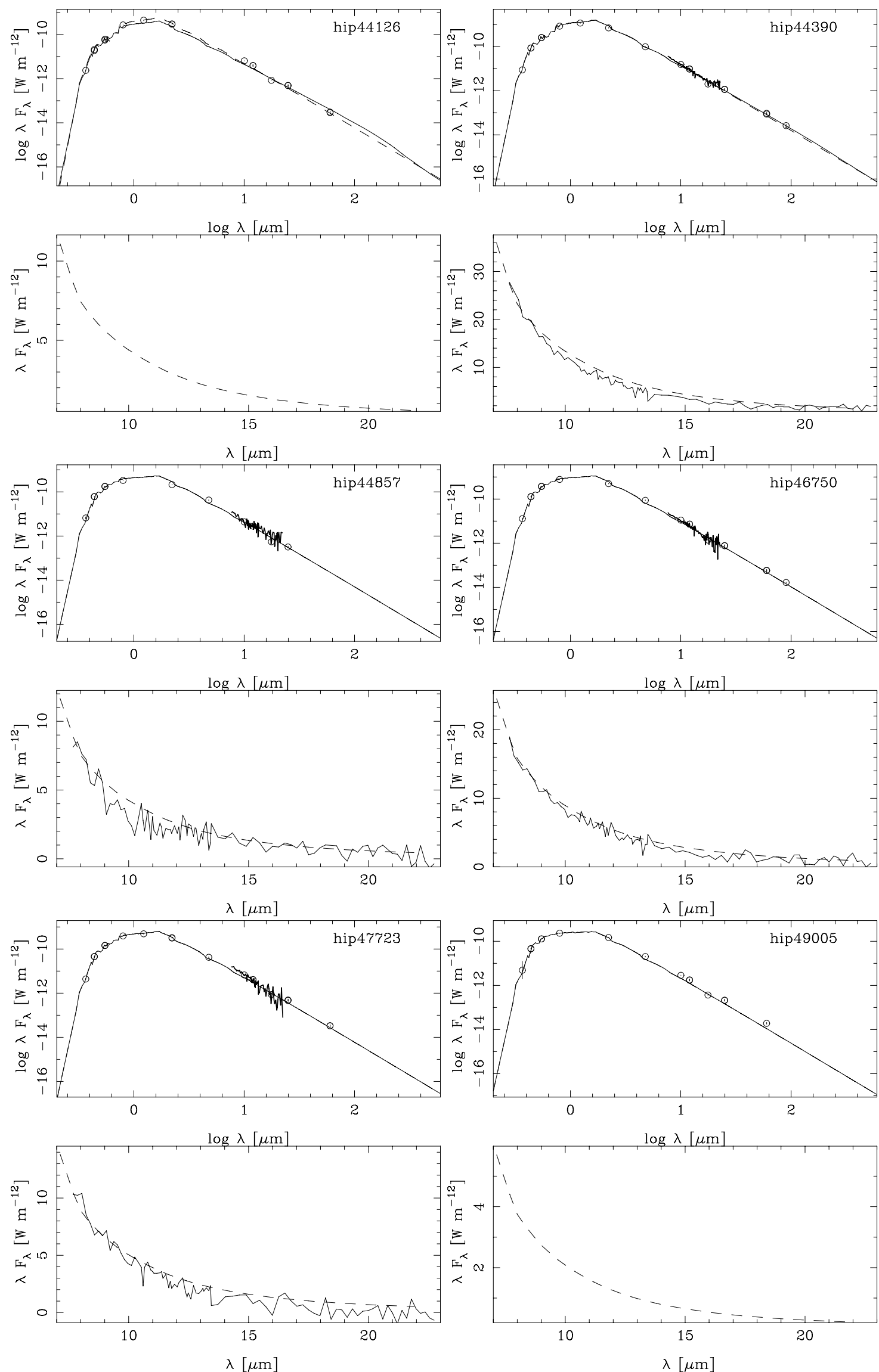

Fig. A.1. continued. 
M. A. T. Groenewegen: Infrared excess around nearby red giant branch stars and Reimers law
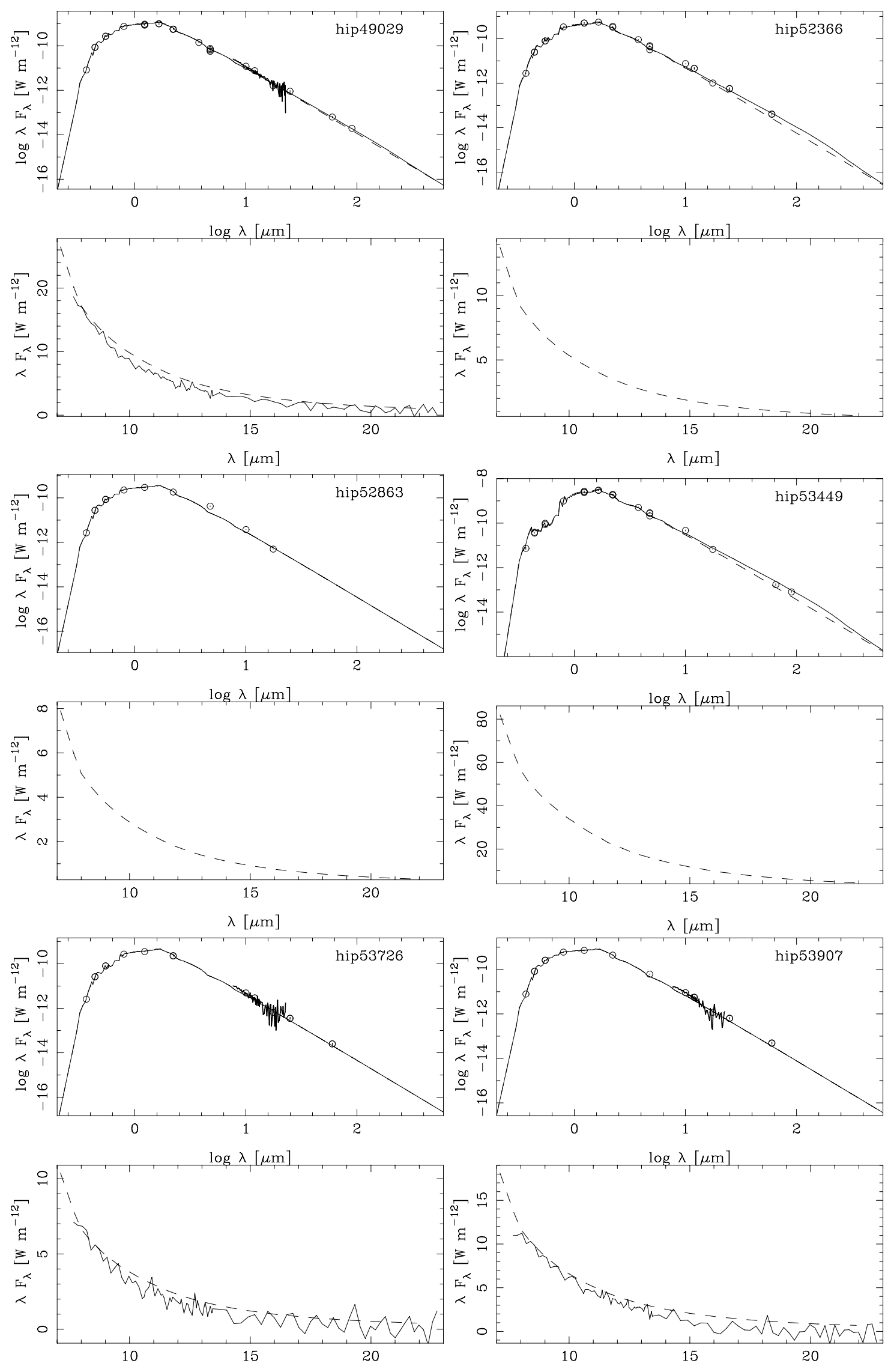

$\lambda[\mu \mathrm{m}]$

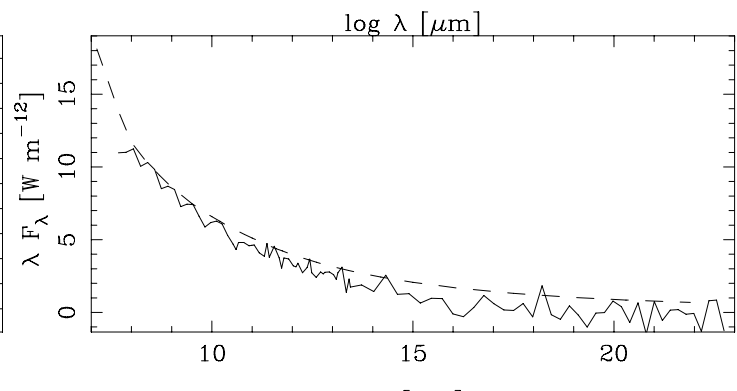

Fig. A.1. continued. 
A\&A 540, A32 (2012)
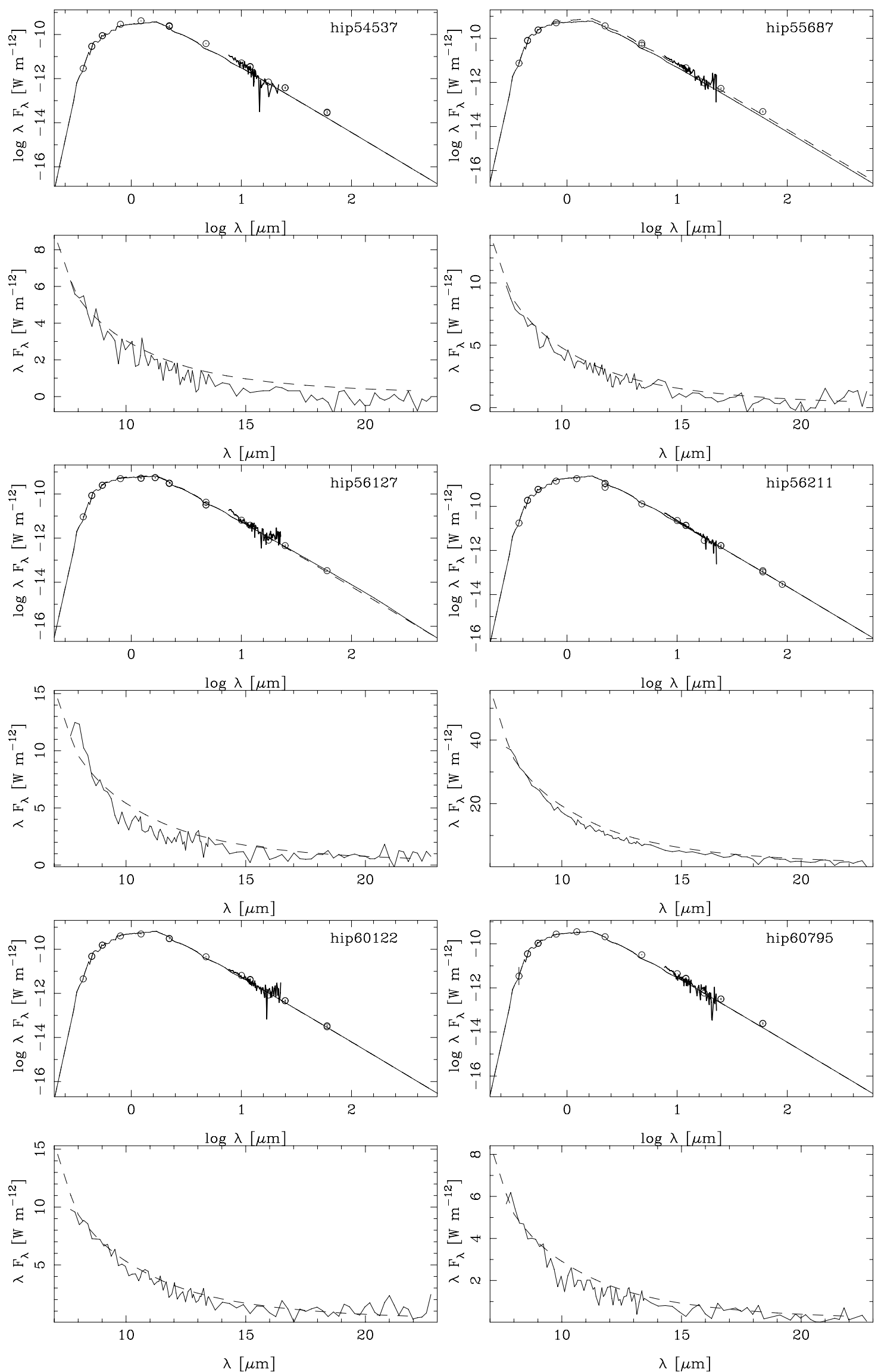

Fig. A.1. continued. 
M. A. T. Groenewegen: Infrared excess around nearby red giant branch stars and Reimers law
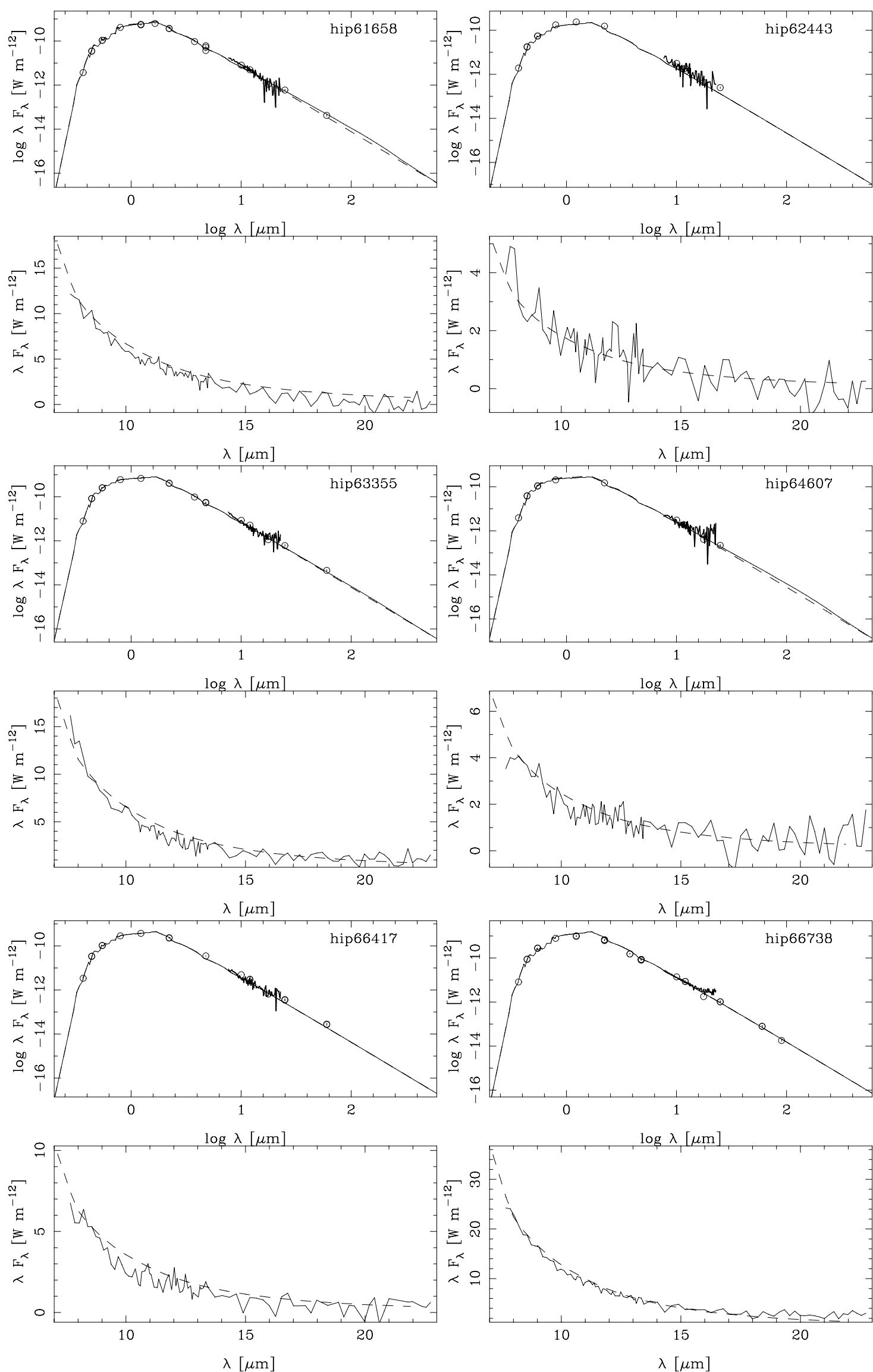

Fig. A.1. continued. 
A\&A 540, A32 (2012)
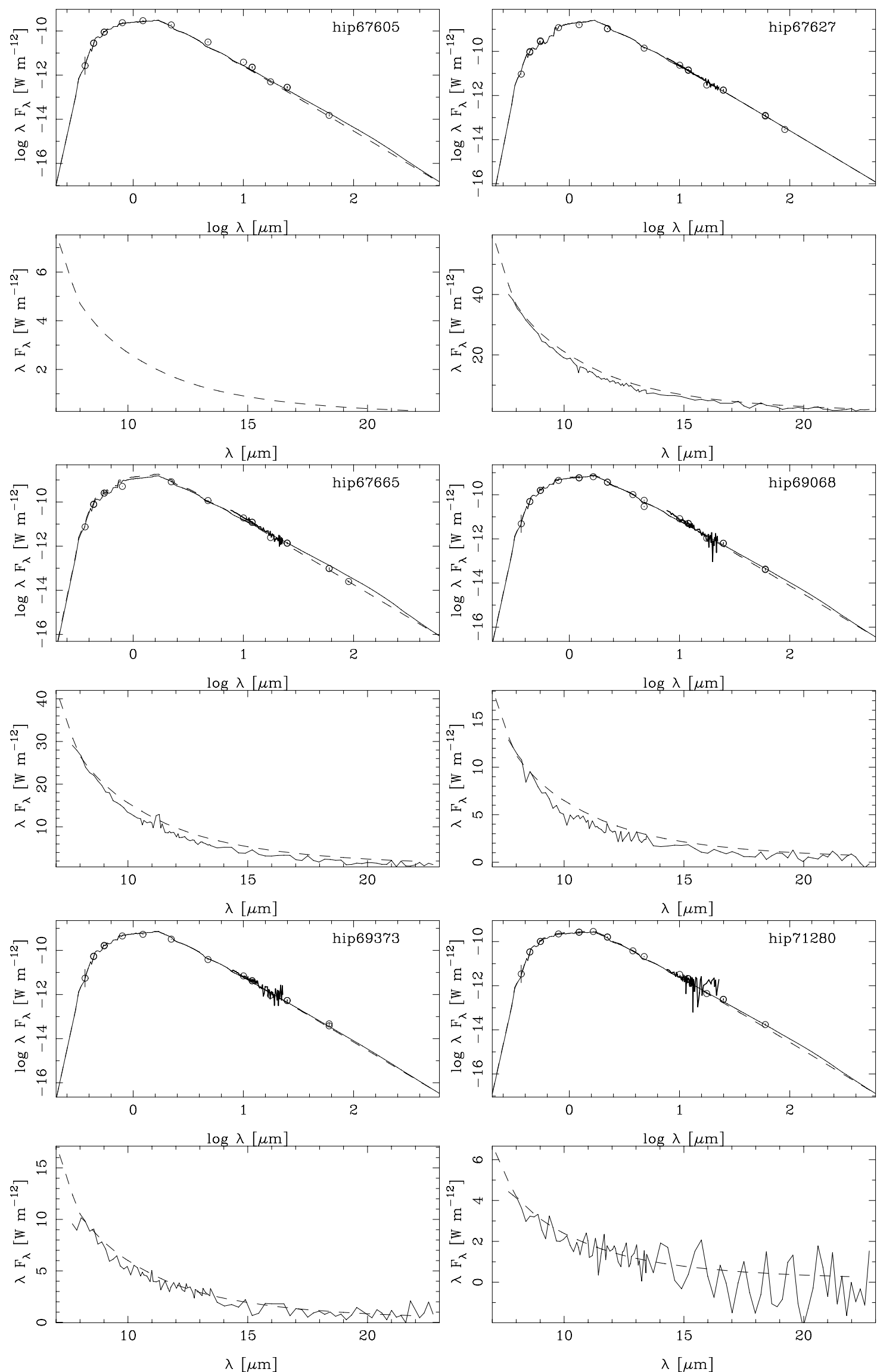

Fig. A.1. continued. 
M. A. T. Groenewegen: Infrared excess around nearby red giant branch stars and Reimers law
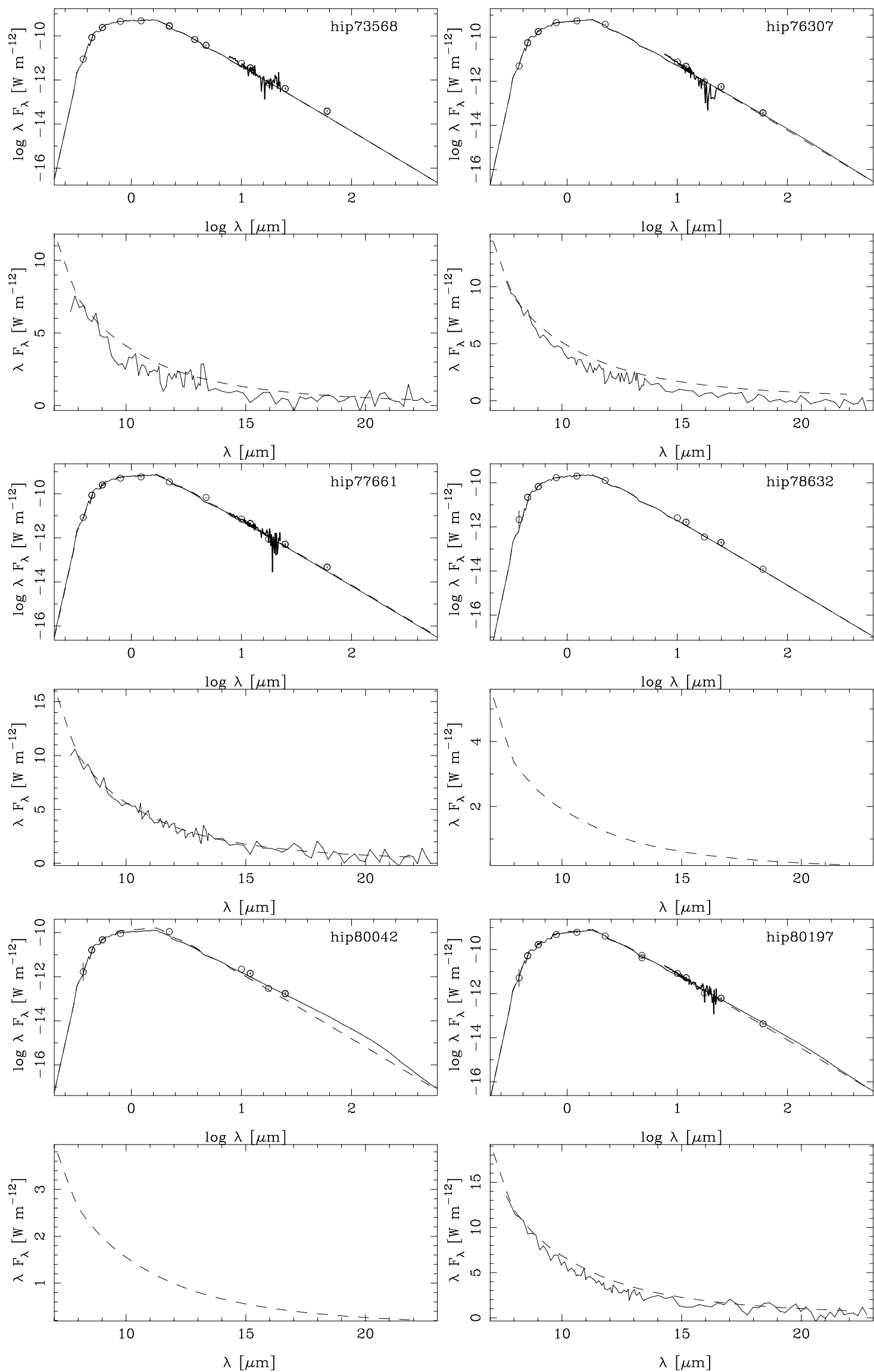

Fig. A.1. continued. 
A\&A 540, A32 (2012)
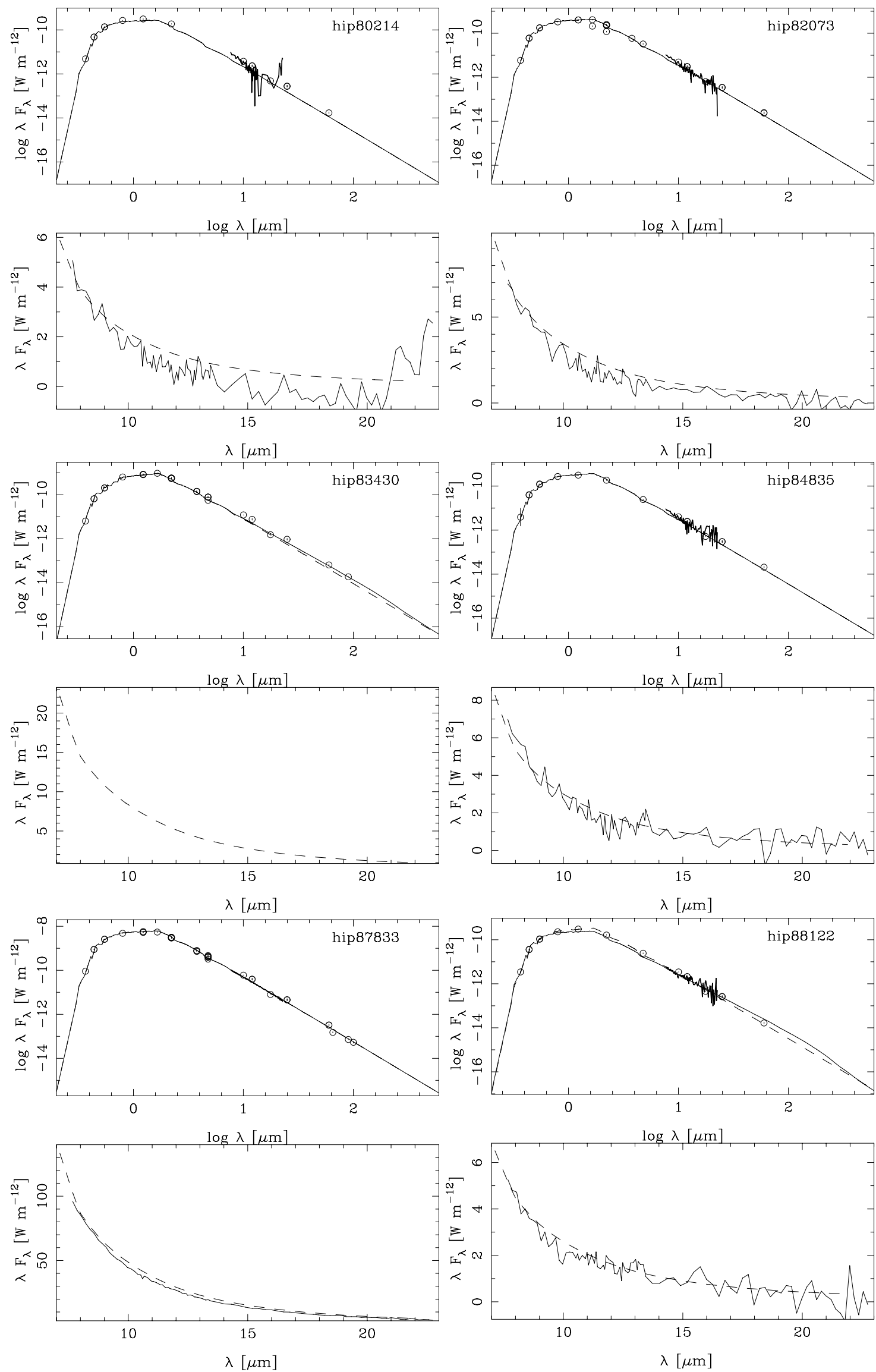

Fig. A.1. continued. 
M. A. T. Groenewegen: Infrared excess around nearby red giant branch stars and Reimers law
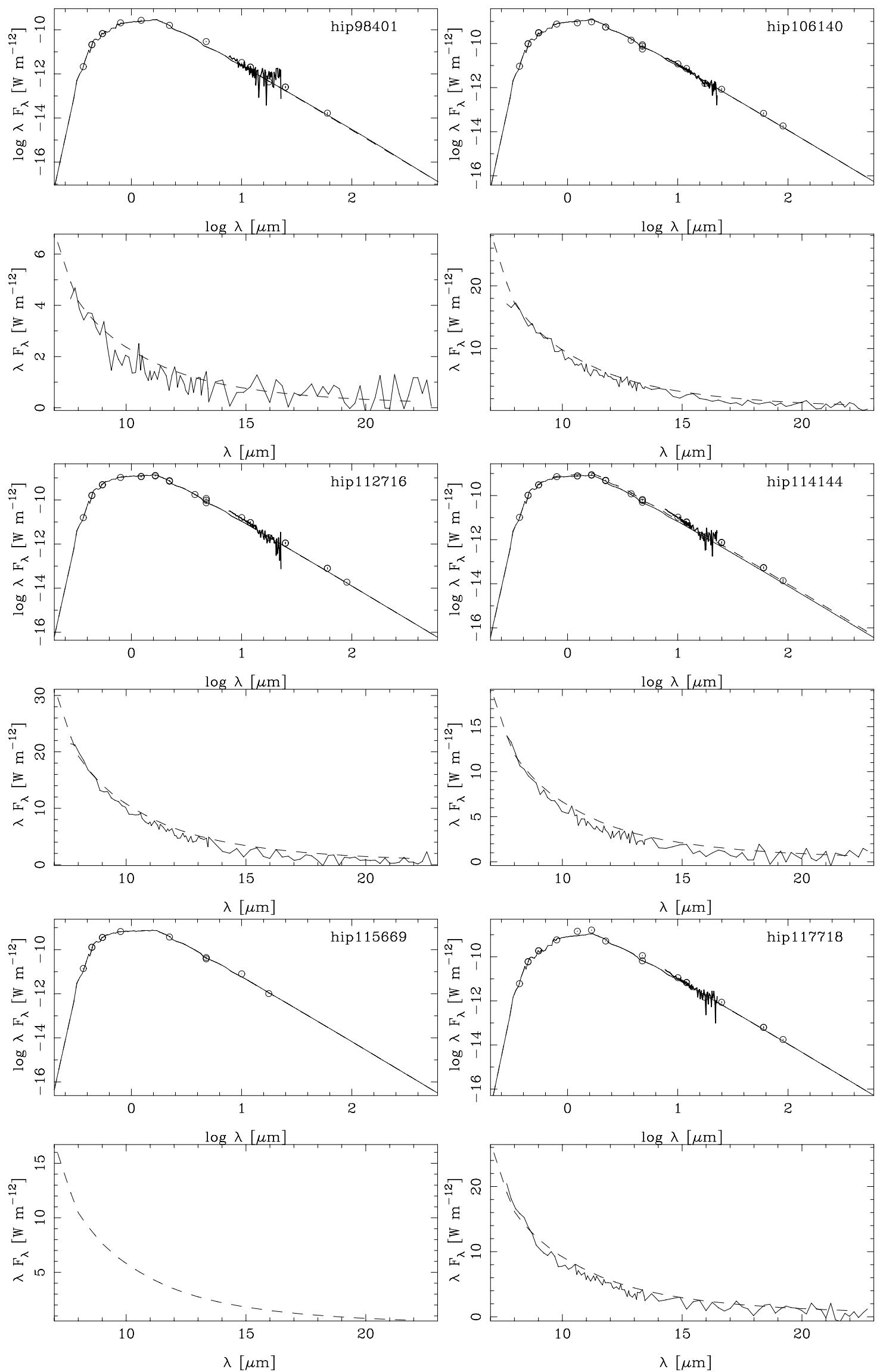

Fig. A.1. continued. 